\title{
An analytical model of interfacial energy based on a lattice-matching interatomic energy
}

\author{
Brandon Runnels ${ }^{\mathrm{a}}$, Irene J. Beyerlein ${ }^{\mathrm{b}}$, Sergio Conti $^{\mathrm{c}}$, Michael Ortiz ${ }^{\mathrm{a}}$ \\ ${ }^{a}$ Division of Engineering and Applied Science, California Institute of Technology, Pasadena, CA 91125, USA \\ ${ }^{b}$ Theoretical Division, Los Alamos National Laboratory, Los Alamos, NM 87545, USA \\ ${ }^{c}$ Institut für Angewandte Mathematik, Universität Bonn, 53115 Bonn, Germany
}

\begin{abstract}
We develop an explicit model for the interfacial energy in crystals which emphasizes the geometric origin of the cusps in the energy profile. We start by formulating a general class of interatomic energies that are reference-configuration-free but explicitly incorporate the lattice geometry of the ground state. In particular, away from the interface the energy is minimized by a perfect lattice. We build these attributes into the energy by locally matching, as best as possible, a perfect lattice to the atomic positions and then quantifying the local energy in terms of the inevitable remaining mismatch, hence the term lattice-matching used to describe the resulting interatomic energy. Based on this general energy, we formulate a simpler rigid-lattice model in which the atomic positions on both sides of the interface coincide with perfect, but misoriented, lattices. In addition, we restrict the lattice-matching operation to a binary choice between the perfect lattices on both sides of the interface. Finally, we prove an $L^{2}$-bound on the interatomic energy and use that bound as a basis for comparison with experiment. We specifically consider symmetric tilt grain boundaries (STGB), symmetric twist grain boundaries (STwGB) and asymmetric twist grain boundaries (ATwGB) in face-centered cubic (FCC) and body-centered cubic (BCC) crystals. Two or more materials are considered for each choice of crystal structure and boundary class, with the choice of materials conditioned by the availability of molecular dynamics data. Despite the approximations made, we find very good overall agreement between the predicted interfacial energy structure and that predicted by molecular dynamics. In particular, the positions of the cusps are predicted correctly, and therefore, although surface reconstruction and faceting are not included in the model, the dominant orientations of the facets are correctly predicted by our geometrical model.
\end{abstract}

\section{Introduction}

Interfaces have long been known to be key players in the mechanics of mesoscopic phenomena. Recrystallization [1], microstructure formation in solidification [2], and microstructure evolution [3], are all examples of phenomena that are significantly influenced by interfacial mechanics. A motivating example of interface-dominated behavior is the manufacture of nanolayered composites by severe plastic deformation, in which it has been shown that at high layer density, layer evolution is dominated by interface mechanics $[4,5]$. Because of the many instances in which interfaces play a significant role, the understanding and prediction of interfacial behavior has elicited considerable interest. However, interfaces stand out among material defects for their variety and complexity, and attempts to characterize them remain ongoing, with approaching ranging from ab-initio quantum-based methods to heuristic geometrical constructions.

The space of interface configurations is quite large, which hinders efforts to identify one unifying characteristic to which the energy of all interfaces can be linked. Consequently, it becomes necessary to distinguish between - and create models for - different interface types. Interfaces between identical crystal lattices are referred to as homophase or grain boundaries (GBs). The simplest non-trivial grain boundaries are twin boundaries, which require no displacement of individual atoms in either lattice to maintain registry at the boundary. Twin boundaries have the highest degree of order possible in an interface and have relatively low 
energy. Many types of high-order boundaries are not twin boundaries, but have a high degree of order and locally minimize the energy. Energy minimizing configurations in the space of interface energy orientations form energy cusps near their location in configuration space [6-8]. Though the interfaces themselves are well-understood, a current challenge is to determine the locations of the cusps, and hence the stability of interfaces in configuration space. The remainder of interface types are low-order boundaries due to the large rotation required to obtain them from the nearest high-order boundary, and quantifying their energy can present a challenge for low-order models.

The experimental measurement of the energy of grain boundaries entails considerable difficulty and there remains a paucity of observational data for validating models of interface energy. Therefore, it is common to resort to atomistic simulations in lieu of experimental data. Density functional theory (DFT) has been used to examine the properties of some grain boundaries and interfaces $[9,10]$. However, the evaluation of general grain boundaries requires consideration of large material samples and is currently prohibitively expensive by DFT. Currently the de facto method for garnering grain boundary energy data is molecular dynamics (MD). The typical computational approach is to create two lattices of atoms, bring them together, apply periodic boundary conditions (or other boundary conditions, c.f. [11]), and relax to static equilibration. Further, with MD, the interface can be locally relaxed or reconstructed to obtain even lower energy structures at the cusp locations [12]. All MD simulations require the use of an empirical interatomic potential. The most commonly used potential in interface calculations is the Embedded Atom Model (EAM) potential [13]. The literature is rich with MD data on a large subset of the five-dimensional configuration space of interfaces. Data for FCC-FCC [14-21], BCC-BCC [22-24], HCP-HCP [25, 26], and FCC-BCC [27] provide solid benchmarks for model verification.

Considerable effort has also been devoted to devising analytical models of interfacial energy. Theories based on dislocation mechanics can be traced back to the original insights of G. I. Taylor [28], whose treatment of dislocation surfaces led to the celebrated work of Read and Shockley [29]. They considered a generic low-angle tilt boundary between two cubic lattices, and used geometry to determine the corresponding dislocation density. By an appeal to the linear-elastic solution for the stress field of an infinite rows of dislocations, they obtained the classic equation for tilt interface energy as a function of tilt angle, $E=E_{0} \theta[A-\ln \theta]$. The model has been shown to work well for low angle tilt, and Wolf demonstrated that the model can be adapted to large-angle tilt boundaries to some degree [30], although this relationship is generally recognized as strictly empirical [31] Read and Shockley's contemporary, J. H. van der Merwe, elucidated the elastic field of symmetric tilt grain boundaries using a periodic interplanar potential [32-34]. In a similar vein, R. Bullough extended the established theory of Peierls-Nabarro [33] for edge dislocations to symmetric tilt boundaries by treating them as arrays of evenly spaced edge dislocations [35]. General representations of interfaces as arrays of dislocations where derived by Frank and Bilby [36, 37], and recent work by van Beers et al was recently published that uses the Frank-Bilby formalism to connect the atomistic description to a continuum model [38]. Despite the insight provided by linear-elastic dislocation models, their ability to predict the cusp structure of interfacial energy for general interfaces is limited owing to the continuum treatment of the crystals.

Another line of inquiry derives from the empirical observation that interfacial energy is largely determined by crystallography. For instance, most face-centered cubic crystals exhibit similar GB anisotropy up to a multiplicative constant [39]. Several models have been proposed that connect the energy of an interface to a geometrically-determined quantity. Perhaps no geometric object is more commonly encountered in interface theory than the coincident site lattice (CSL). The ratio of the area of the CSL unit cell to the area of the lattice unit cell gives the so-called $\Sigma$ value of the interface. Because low $\Sigma$ values correspond, in general, to low energy grain boundaries, the CSL has been proposed as a model for GB energy [40, 41]. However the validity of such models has been questioned [42] and, to date, there is no general consensus regarding their reliability for general grain boundaries [43]. The structural unit model provides an alternative geometric approach [44-48]. The structural unit model is a heuristic method that attempts to characterize interfaces by determining the requisite structures needed for-or resulting from - compatibility between the crystals. The model is helpful in understanding the geometry of unstructured interfaces, but it does not provide a means for predicting GB energy. A similar construction is the O-lattice of Bollmann, which provides a geometrical measure of the number of coincident points, or $O$-points, in two interpenetrating lattices. From 
this measure, the degree of registry and, ostensibly, the dislocation content of the interface may be inferred [49]. By using geometric constructions of these types and assuming the existence of ordered networks of interfacial dislocations, it is possible to use anisotropic elasticity theory to compute the resultant energy as well as the full stress field [50]. However we are not aware of any procedure for predicting the location of energy cusps for arbitrary interfaces by means of these methods.

Another vein of research has been to model energy of arbitrarily oriented grain boundaries by using physical models for large-angle grain boundaries and matching the results to known cusp locations. One such approach is to treat the defects at large-angle grain boundaries as disclinations. This methodology was introduced by Li [51] and extended by Shih [52], Nazarov [53], and recently by Fressengeas [54]. An additional approach based on the concept of "grofs" was recently introduced by Bulatov et al to model large-angle grain boundaries between known cusps. [55]. Models of this variety are able to capture a wide range of grain boundary behavior, but require a priori knowledge of the energy cusp locations.

The purpose of the present work is the development of a model capable of quickly characterizing the structure of the interfacial energy for arbitrary interfacial geometries, especially the location of the energy cusps. In order to elucidate this connection, we start by developing a general representation of the energy of an assembly of atoms suitable for configurations which are only partially crystalline. Our interatomic energy is reference-configuration-free, in order not to privilege one special orientation of the crystal lattice and to deal seamlessly with small and large-angle grain boundaries. At the same time, it is important that the interatomic energy explicitly incorporates the properties of the ground state. In particular, away from the interface the energy should be minimized by a perfect lattice. We build these attributes into the energy by locally matching, as best as possible, a perfect lattice to the atomic positions and then quantifying the local energy in terms of the inevitable remaining mismatch, hence the term lattice-matching used to describe the resulting interatomic energy. We first formulate the interatomic energy for the case in which the ground state is sure to correspond to one single crystal class and then show how it can be generalized to include multiple crystal phases. This extension enables the study of interfaces between different variants of a material, such as result from solid-solid phase transitions, and of interfaces between dissimilar crystals. Our interatomic energy is inspired by the work of Luckhaus and Mugnai [56], see also [57]. However, our energy is different from that of Luckhaus and Mugnai [56] in that it is completely discrete and does not include a continuum elastic energy.

In order to facilitate the analysis of interfacial energy, we formulate a simpler rigid-lattice model in which the atomic positions on both sides of the interface coincide with perfect, but misoriented, lattices. In this rigid-lattice model, the positions of the atoms in the vicinity of the interface are not relaxed to their equilibrium positions. In addition, we restrict the lattice-matching operation to a binary choice between the perfect lattices on both sides of the interface. Finally, we prove an $L^{2}$-bound on the interatomic energy and use that bound as a basis for comparison with experiment. We specifically consider homophase interfaces, or grain boundaries, i. e., interfaces at which the abutting lattices differ by a rotation. We consider the following three types of interfaces: symmetric tilt grain boundaries (STGB), symmetric twist grain boundaries (STwGB) and asymmetric twist grain boundaries (ATwGB). Additionally, we consider materials whose lattice structure falls into one of three classes: face-centered cubic (FCC), body-centered cubic (BCC) and hexagonal close-packed (HCP). Two or more materials are considered for each choice of crystal structure and boundary class, with the choice of materials conditioned by the availability of molecular Despite the approximations made, we find very good overall agreement between the predicted interfacial energy structure and that predicted by molecular dynamics. In particular, the positions of the cusps are predicted correctly.

\section{Theory}

The specific aim of the present work is to characterize the excess free energy of infinite planar interfaces separating two semi-infinite crystals. The essence of the approach is to model the interface using an atomistic method, with the motivation that registry across an interface appears to correlate to low-energy interfaces. The key result is an integral formulation of the total energetic cost of deformation of a lattice from one configuration to another across the interface. 
A primary feature of the end result is a highly reduced order model with only macroscopic degrees of freedom. A number of simplifications and approximations must be made. The primary simplification is that we do not account for atomistic-level phenomena such as the formation of misfit dislocations/eigenstrains or local/sub-lattice atomistic relaxations (c.f. [58]). The driving factor in our energy anisotropy functional is, ultimately, the lattice matching on either side of the interface. The implication of this assumption is that other interfacial mechanisms are driven by geometric atomic registry.

Our key idea is that the interface is the boundary between two different crystal structures (possibly, two different orientations of the same lattice). Each of them is "in the bulk" energy-minimizing, but close to the interface the energy will be higher (Section 2.1). In order to treat the two sides symmetrically, we refrain from chosing a reference configuration, and use sets of points (the atomic positions) as the basic variables. The energy of the inteface arises from the fact that the atoms close to it have neigbours in the "wrong" positions. Therefore the additional energy arises from the displacement of the neighbors with respect to the "ideal" positions in the perfect lattice (see Section 2.2). We first use a localization function to select the range of interaction $\lambda$, and then write the energy as a sum of the "cost" of deforming each local configuration to the one that would appear in a perfect lattice, as done in (7) below. Physically this means that the energy density around a point $z \in \mathbb{R}^{3}$ depends on the positions of all atoms located at distance less then $\lambda$ from $z$, and vanishes if these positions lie on the perfect lattice, as illustrated in Figure 2 below. Of course, in the process one needs to optimize over all possible isometric translations of the lattice, which all have the same energy, resulting in the minimization over translations $\tau$ and rotations $R$ in (7). In Section 2.3 we include temperature by replacing the sharp atomic positions by "clouds" with a temperature-dependent length scale, whose profile is given by the function $\psi_{\beta}$, and simplify the numerical evaluation of the model by replacing it by an $L^{2}$ bound (Sect. 2.4).

The development of this section proceeds as follows. We begin by establishing, for reference, the notions of total energy and excess free energy. We then introduce the notion of "lattice-matching" as a means of determining the total energetic cost of deforming a lattice from its closest perfect configuration, making precise the notion of a "reference lattice" in the context of energy minimization. Subsequently, we introduce the notion of thermalization as a means of obtaining an easily computable bound of the grain boundary energy. The result of the subsection on thermalization is an expression for the model in its most general form; this form is specialized and reduced in the following section to enable computation.

\subsection{Formal definition of excess free energy}

We begin by considering two clusters of atoms, labeled $b$ and $w$ ("black" and "white"), possibly of different species, interacting through a general interatomic energy. Without approximation, the resulting total energy can be formally written as some function $E_{\text {tot }}\left(\chi^{b}, \chi^{w}\right)$ of the black and white atomic coordinates at equilibrium, respectively. When considering infinite systems, direct evaluation of $E_{\text {tot }}\left(\chi^{b}, \chi^{w}\right)$ results in infinite energies in general. However the interfacial energy, or excess energy density per unit area of interface, may be expected to be well defined.

To begin, we review the formulation of general surface energy in a continuum setting. We note that care must be taken in determining the correct derivation and form of surface energy [59], and we begin with an integral formulation for the total free energy of a general incoherent interface as defined using the Gibbs dividing surface framework (c.f. [60]).

$$
E_{\mathrm{tot}}=\int_{\Omega^{b}} E^{b}(x) d x+\int_{\Omega^{w}} E^{w}(x) d x+\int_{\Omega^{b} \cap \Omega^{w}} \gamma(x) d \mathcal{H}^{2},
$$

where $\Omega^{b}=[-L / 2, L / 2]^{2} \times[-H / 2,0], \Omega^{w}=[-L / 2, L / 2]^{2} \times[0, H / 2]$ with $\Omega^{b} \cap \Omega^{w}$ contained in the interface, $E^{b}, E^{w}$ are the intensive (per unit volume) free-energy densities in $b$ and $w$, respectively, and $\gamma$ is the interfacial excess free energy. We isolate the above expression for $\gamma$ in the following way. The free energy expressions $E^{b}, E^{w}$ evaluated far from the interface are expressed as

$$
\int_{\Omega^{b, w}} E^{b, w}(x) d x=N_{L, H}^{b, w} E_{\mathrm{coh}}^{b, w}
$$




\begin{tabular}{ll}
\hline Notation & Description \\
\hline$\beta$ & Reciprocal temperature $\left(1 / k_{B} T\right)$ \\
$B_{\lambda}(z)$ & Ball of radius $\lambda$ centered at point $z \in \mathbb{R}^{3}$ \\
$C_{k}(X, Y)$ & Cost function of mapping $k$ atoms from configuration $X$ to configuration $Y$ \\
$C(X, Y, z)$ & Thermalized cost function \\
$C^{\beta}(X, Y, z)$ & $C(X, Y, z)$ with atoms at temperature $\beta$ \\
$e(\chi, z)$ & Local cost of mapping a deformed lattice $(\chi)$ to its reference lattice \\
$e^{\beta}(\chi, z)$ & $e(\chi, z)$ for lattice at temperature $\beta$ \\
$E(\chi, \Omega)$ & Total cost (integrated over $\Omega)$ of mapping $\chi$ to its reference lattice \\
$E^{\beta}(\chi, \Omega)$ & $E^{\beta}(\chi, \Omega)$ with atoms at temperature $\beta$ \\
$g(|x-y|)$ & Cost of mapping $x$ to $y$ \\
$\gamma$ & Interfacial energy per unit area \\
$\mathcal{L}$ & Collection of reference lattice coordinates \\
$\phi$ & Mapping between lattice configurations \\
$R \in S O(3)$ & Rotations in $\mathbb{R}^{3}$ \\
$\rho_{\beta}^{X}(x)$ & Effective density function for atoms in configuration $X$ at temperature $\beta$ \\
$\theta(x)$ & Localization function on a length scale $\lambda$ \\
$\psi_{\beta}(x)$ & Thermalization mollifier for reciprocal temperature $\beta$ \\
$X, Y, \chi=\left\{x_{0}, x_{1}, \ldots\right\} \subset \mathbb{R}^{3}$, & Sets of atomic coordinates \\
$\Omega \subset \mathbb{R}^{3}$ & Domain of consideration \\
$A_{k}$ & The family of $k$-tuples of points on $\mathbb{R}^{3}$ \\
$\# a$ & The number of elements of the set $a$ \\
\hline
\end{tabular}

Table 1: Nomenclature

where $N_{L, H}^{b, w}$ are the number of atoms contained in $\Omega^{b, w}$, and $E_{\text {coh }}^{b, w}$ is the cohesive energy per atom at a distance sufficiently far from the interface so as to not be affected by it. (We note that the notion of "sufficiently far" varies from material to material; in general we consider metallic interfaces where the interfacial length scale is on the order of several lattice spacings.) Additionally we express the averaged excess energy as

$$
\int_{\Omega^{b} \cap \Omega^{w}} \gamma(x) d \mathcal{H}^{2}=\gamma L^{2}
$$

Then we obtain an expression for the interfacial energy by rearranging and taking the limit (cf. [31, 61-63])

$$
\gamma=\lim _{L \rightarrow+\infty} \frac{1}{L^{2}}\left(\lim _{H \rightarrow+\infty}\left(E_{\mathrm{tot}}\left(\chi_{L, H}^{b}, \chi_{L, H}^{w}\right)-N_{L, H}^{b} E_{\mathrm{coh}}^{b}-N_{L, H}^{w} E_{\mathrm{coh}}^{w}\right)\right)
$$

where $E_{\mathrm{tot}}\left(\chi_{L, H}^{b}, \chi_{L, H}^{w}\right)$ is the total energy at equilibrium, $E_{\mathrm{coh}}^{b, w}$ are the cohesive energies per atom in infinite crystals of type $b, w$ respectively, and $N_{L, H}^{b, w}$ are the corresponding numbers of atoms in each subsystem, respectively. Provided that the limits are well-defined, and that the equilibrium configuration of the atoms away from the interface corresponds to two perfect - albeit misoriented-lattices, eq. (4) returns the interfacial energy per unit area of an infinite planar interface. The aim of the remainder of the paper is to characterize $\gamma$ explicitly as a function of the interface geometry.

\subsection{A lattice-matching interatomic energy}

In this section we present a general model for the energy of a set of atoms which does not appeal to a reference configuration. In the next sections we shall present a simpler expression, derived in a mathematically precise way as an upper bound of interfacial energy. While we adopt standard convention wherever 
possible, specific nomenclature occasionally necessary. In addition to their definitions throughout the text, conventions and symbols are enumerated in Table 1.

We begin by formulating a representation of the interatomic energy of an arbitrary ensemble of atoms $\chi=\left\{x_{0}, x_{1}, \ldots\right\} \subset \mathbb{R}^{3}$ that builds in explicitly the property that the ground state of a countable set of atoms be a prespecified crystal lattice. The proposed interatomic energy applies directly to atoms in arbitrary positions and does not make use of a specific reference configuration or neighbouring relations. At the same time, the model energy explicitly assumes that the ground state is locally crystalline, in the sense that, locally, the energy density is minimized if the atomic positions coincide, up to a rigid-body motion, with the positions specified by a reference lattice $\mathcal{L}$. For example, for a bcc crystal $\mathcal{L}_{\mathrm{bcc}}=a \mathbb{Z}^{3} \cup\left(a \mathbb{Z}^{3}+\left(\frac{a}{2}, \frac{a}{2}, \frac{a}{2}\right)\right)$ and similarly for other crystal classes. Consequently, the result of the lattice-matched energy ostensibly includes contributions from relaxation mechanisms such as misfit dislocations or sub-lattice relaxation. This construction is useful not only for quantifying the deformation energy, but also for making precise the notion of a reference lattice.

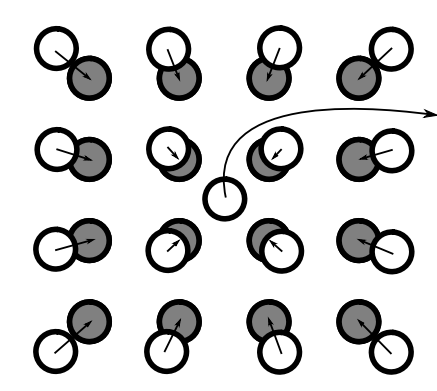

Figure 1: The cost functional measures the energetic cost of deforming an imperfect lattice (e.g. a lattice with an interstitial atom-white) to a perfect lattice (gray). Note that atoms can be "gained" or "lost" (e.g. in the figure, the interstitial atom is lost) as a consequence of locality. The creation of an interstitial can be seen as the process of "bringing in" an atom from infinity.

We start by quantifying the difference between two configurations $X, Y \subset \mathbb{R}^{3}$ of atoms via a measure of the cost incurred in deforming one onto the other. To make this measure independent of the labeling of the atoms we minimize over all possible bijective maps $\varphi: X \rightarrow Y$, which correspond to all possible relabellings of atoms. We consider general multibody interactions, and write a generic transportation cost as a cluster expansion. For $k \geq 1$, let $A_{k}=\left\{a \subset \mathbb{R}^{k}: \# a=k\right\}$ be the family of sets of $k$ points in $\mathbb{R}^{3}$, or $k$-clusters, and let $C_{k}: A_{k} \times A_{k} \rightarrow[0, \infty)$ be the cost of deforming the $k$ atoms $\left\{x_{1}, \ldots, x_{k}\right\}$ into the positions $\left\{y_{1}, \ldots, y_{k}\right\}$. For instance, $C_{1}\left(\left\{x_{1}\right\},\left\{y_{1}\right\}\right)$ measures the cost of transporting the singleton $\left\{x_{1}\right\}$ to a new position $\left\{y_{1}\right\}, C_{2}\left(\left\{x_{1}, x_{2}\right\},\left\{y_{1}, y_{2}\right\}\right)$ measures the cost of transporting the pair of points $\left\{x_{1}, x_{2}\right\}$ to a new configuration $\left\{y_{1}, y_{2}\right\}$, and so on. We assume $C_{k}$ to be symmetric and invariant under isometries, in the sense that $C_{k}(a, b)=C_{k}(b, a)$ and for any affine isometry $I: \mathbb{R}^{3} \rightarrow \mathbb{R}^{3}$,

$$
C_{k}(I(a), I(b))=C_{k}(a, b)
$$

Locality is enforced through a localization function $\theta \in C_{c}^{\infty}\left(\mathbb{R}^{3}\right)$. This localization function is positive in a neighborhood of the origin larger than the lattice spacing and vanishes outside of a ball whose radius $\lambda$ is larger than the lattice spacing.

Here and subsequently, given $a \in A_{k}$ and a function $f: \mathbb{R}^{3} \rightarrow \mathbb{R}^{3}$, we define $f(a) \in A_{k}$ as $f\left(\left\{x_{1}, \ldots, x_{k}\right\}\right)=$ $\left\{f\left(x_{1}\right), \ldots, f\left(x_{k}\right)\right\}$. The local cost of deformation in the neighborhood of a point $z \in \mathbb{R}^{3}$ is given by summing the cost of all clusters up to order $K$, with the result,

$$
C(X, Y, z)=\inf \left\{\sum_{k=1}^{K} \sum_{a \subset X: \# a=k} C_{k}(a, \varphi(a)) \prod_{x \in a}(\theta(x-z)+\theta(\varphi(x)-z)), \varphi: X \rightarrow Y \text { bijective }\right\}
$$


Both $X$ and $Y$ are assumed to be countable. The map $\varphi$ only needs to be defined in a neighborhood of $z$ defined by the support of the localization function $\theta$. Indeed, the condition " $\varphi$ bijective" can be replaced by " $\varphi$ injective on $X \cap B_{\lambda}(z)$ and surjective on $Y \cap B_{\lambda}(z)$ ", which means that if $x, x^{\prime} \in X \cap B_{\lambda}(z)$ and $x \neq x^{\prime}$, then $\varphi(x) \neq \varphi\left(x^{\prime}\right)$ and, for any $y \in Y \cap B_{\lambda}(z)$, there is $x \in X$ with $\varphi(x)=y$. We note that, by virtue of its definition, the cost function $C$ is symmetric, i. e., $C(X, Y, z)=C(Y, X, z)$. The localization operation is devised so as to include all atoms whose position in either the $X$ or the $Y$ configuration is close to $z$. In particular, The localization operation permits consideration of interstitial atoms and vacancies, cf. Fig. 1.

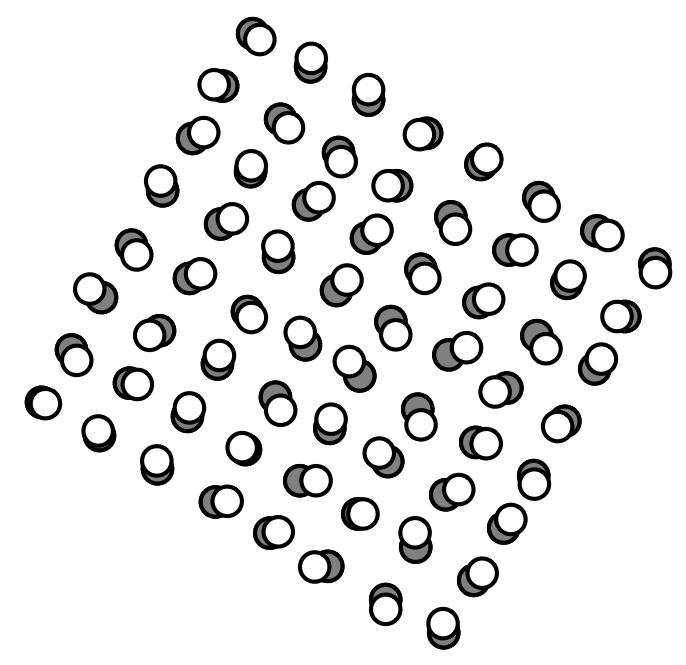

Figure 2: The orientation of the local reference lattice (gray) for an arbitrarily deformed lattice (white) is found by minimizing the cost function with respect to all rigid-body translations.

We now identify the local energy density as the cost of local deformation to the perfect lattice. However, in order to take into account the possible orientations and translations of the lattice, in computing the local energy density we minimize over all isometries, cf. Fig. 2, with the result

$$
e(\chi, z)=\inf \left\{C(\chi, R \mathcal{L}+\tau, z): R \in \mathrm{SO}(3), \tau \in \mathbb{R}^{3}\right\}
$$

This optimization may be regarded as local lattice-matching, in the sense of identifying the perfect lattice that best fits locally the atomic positions. If there are multiple possible lattices, labeled by an index $i \in I$, we additionally minimize over the choice of lattice, with the result

$$
e(\chi, z)=\inf \left\{C\left(\chi, R \mathcal{L}_{i}+\tau, z\right): R \in \mathrm{SO}(3), \tau \in \mathbb{R}^{3}, i \in I\right\}
$$

Finally, we integrate the local energy density over a domain $\Omega \subset \mathbb{R}^{3}$, to obtain the total energy

$$
E(\chi, \Omega)=\int_{\Omega} e(\chi, z) d z
$$

We remark that for $\chi=\mathcal{L}$, it follows that $e(\mathcal{L}, z)=0$ for all $z$ and, correspondingly, $E\left(\mathcal{L}, \mathbb{R}^{3}\right)=0$, which ensures that the perfect lattice $\mathcal{L}$ is indeed the ground state of the energy.

We stress that at this stage the model is very general and includes the possibility of surface reconstruction, faceting, any much else. In particular, the same approach could be used to study a localized inclusion (a small grain contained in a single crystal) or also a small particle (a small grain, or polycrystal, surrounded by vacuum). The practical implementation, however, is computationally demanding, and it is also not clear how to efficiently parametrize the cost functions $C_{k}$ with few parameters. 


\subsection{Thermalization and $L^{2}$ bound}

We proceed to extend the preceding model energy so as to take temperature into account. Consideration of temperature is important as the energy and morphology of interfaces is known to be temperature dependent [64]. Thermalization has the additional mathematical consequence of enabling the approximation of the energy by an easy-to-compute $L^{2}$-bound. For simplicity, we consider one-point clusters, $K=1$, only. By invariance under isometries the cost function $C_{1}: \mathbb{R}^{3} \times \mathbb{R}^{3} \rightarrow[0, \infty)$ can only depend on the distance between its arguments, $C_{1}(x, y)=g(|x-y|)$. The simplest example is $C_{1}(x, y)=c|x-y|^{2}$, for some $c>0$. The local cost then reduces to

$$
C(X, Y, z)=\inf \left\{\sum_{x \in X} g(|x-\varphi(x)|)(\theta(x-z)+\theta(\varphi(x)-z)), \varphi: X \rightarrow Y \text { bijective }\right\} .
$$

Within this simplified framework, for temperatures below the melting point, we account for temperature simply by smoothing the positions of the atoms. As long as the time-averaged thermal fluctuations may be assumed to be independent, we determine the smoothing function using the Max-Ent formalism [65-67].

We begin by interpreting the infimum over all bijective maps $\varphi$ as the transport problem,

$$
\inf _{\varphi} \sum_{x \in X} g(|x-\varphi(x)|)(\theta(x-z)+\theta(\varphi(x)-z))=\inf _{T} \int_{\mathbb{R}^{3}} g(|x-y|)(\theta(x-z)+\theta(y-z)) d T(x, y)
$$

where $T \in \mathcal{M}\left(\mathbb{R}^{3} \times \mathbb{R}^{3}\right)$ is a nonnegative measure with marginals $\mu^{X}=\sum_{x \in X} \delta_{x}$ and $\mu^{Y}=\sum_{y \in Y} \delta_{y}$. We then replace the discrete measures by smooth ones. To this end, let $\psi_{\beta} \in C_{c}^{\infty}\left(\mathbb{R}^{3}\right)$ be a mollifier with width depending on temperature. We then set $\rho_{\beta}^{X}=\mu^{X} * \psi_{\beta}$, so that $\rho_{\beta}^{X}(z)=\sum_{x \in X} \psi_{\beta}(x-z)$ is the effective density of the set of atoms $X$ at point $z, \rho_{\beta} \in C^{\infty}\left(\mathbb{R}^{3}\right)$, and replace the local energy cost of deformation $C$ by

$$
C^{\beta}(X, Y, z)=\inf \left\{\int_{\mathbb{R}^{3} \times \mathbb{R}^{3}} g(|x-y|)(\theta(x-z)+\theta(y-z)) d T(x, y): \Pi_{1} T=\rho_{\beta}^{X} d x, \Pi_{2} T=\rho_{\beta}^{Y} d y\right\}
$$

where $\Pi_{1}$ and $\Pi_{2}$ denote the marginals. The thermalized local energy density $e^{\beta}(\chi, z)$ then follows as the least cost of deforming the atomic positions into a perfect lattice, as in (7), and, finally, the total thermalized energy $E^{\beta}(\chi, \Omega)$ is obtained by integration of the local energy density over the domain $\Omega$.

For an efficient evaluation of the thermalized local cost of deformation $C^{\beta}$ it is useful to rewrite it as an integral over $\mathbb{R}^{3}$ rather than $\mathbb{R}^{3} \times \mathbb{R}^{3}$. From a technical viewpoint, we remark that for $g(t)=t^{2}$ we can identify $C^{\beta}$ as having the mathematical form of a 2-Wasserstein distance which behaves, for small deviations, like the squared $H^{-1}$ norm [68, Th. 7.26]. In general, the 2-Wasserstein distance is bounded by the $L^{1}$ norm [68, Prop. 7.10] and, therefore, by the $L^{2}$ norm. However, these abstract results do not have the optimal scaling in the present regime. Further, the general results do not treat the case of a symmetric localization that is important for us, and are therefore not used. We instead prove (see Appendix A) a specific bound via the localized squared $L^{2}$ norm, adapting those general ideas to the present context but without using the concept of Wasserstein distance or $\mathrm{H}^{-1}$.

The bound from Lemma 1 in Appendix A shows that such a reduction of the dimensionality of the integration domain is indeed possible. Specifically, suppose that the mollified density is uniformly bounded from above and below,

$$
\frac{1}{c} \leq \rho_{\beta}^{X}(z) \leq c \text { for all } z \in \mathbb{R}^{3},
$$

and the same for $\rho_{\beta}^{Y}$. These bounds hold, e. g., if the support of $\psi_{\beta}$ is larger than the lattice spacing. Then, the 2-Wasserstein distance is bounded by the squared $L^{2}$ distance. This bound still holds true for the weighted distance under use, which includes the localization function $\theta$. Assuming that $\theta$ is monotonically decreasing in the radial direction, we have (cf. Lemma 1 in Appendix A)

$$
C^{\beta}(X, Y, z) \leq c \int_{\mathbb{R}^{3}}\left|\rho_{\beta}^{X}(x)-\rho_{\beta}^{Y}(x)\right|^{2} \theta(x-z) d x
$$


and, therefore, the local energy density admits the bound

$$
e(\chi, z) \leq \inf \left\{c \int_{B_{\lambda}(z)}\left|\rho_{\beta}^{\chi}(x)-\rho_{\beta}^{R \mathcal{L}+\tau}(x)\right|^{2} \theta(x-z) d x: R \in \mathrm{SO}(3), \tau \in \mathbb{R}^{3}\right\},
$$

with the corresponding bound on the total energy obtained by integration over $\Omega$.

The simple bound (15) provides the basis for the comparison between theory and experiment presented in Section 3.

\subsection{Interfacial energy}

We proceed to specialize the general energy to a flat interface of unit normal $\nu \in S^{2}$ between two copies of a lattice with different orientation. We denote by $\mathcal{L}^{b}, \mathcal{L}^{w}$ the two copies of the lattice, which are given by

$$
\mathcal{L}^{b}=R^{b} \mathcal{L}+\tau^{b} \text { and } \mathcal{L}^{w}=R^{w} \mathcal{L}+\tau^{w}
$$

for some rotations $R^{b}, R^{w} \in \mathrm{SO}(3)$ and translations $\tau^{b}, \tau^{w} \in \mathbb{R}^{3}$. In comparisons with experiment, we specifically consider a rigid lattice model, i. e., we neglect the local relaxation of the atoms in the vicinity of the interface. Under this simplifying assumption, the atomic configuration is then given by

$$
\chi=\left(\mathcal{L}^{b} \cap \mathbb{R}_{b}^{3}\right) \cup\left(\mathcal{L}^{w} \cap \mathbb{R}_{w}^{3}\right),
$$

where

$$
\mathbb{R}_{b}^{3}=\left\{x \in \mathbb{R}^{3}: x \cdot \nu>0\right\} \text { and } \mathbb{R}_{w}^{3}=\left\{x \in \mathbb{R}^{3}: x \cdot \nu<0\right\},
$$

with $\Sigma=\left\{x \in \mathbb{R}^{3}: x \cdot \nu=0\right\}$ the interface. Physically this means that we are using the lattice $\mathcal{L}^{b}$, with orientation given by the rotation $R^{b}$, above the interface, and the other one below it, with a sharp, flat interface. In particular, at this stage surface reconstruction and faceting are excluded from the kinematics. We note that the rigid lattice approximation supplies an upper bound on the local energy density.

In order to simplify the energy further, we assume that the local rotation and translation at a point $z \in \mathbb{R}^{3}$ is the one of the reference lattice around $z$. This means that, for $z \in \mathbb{R}_{b}^{3}$,

$$
e(\chi, z)=\inf \left\{\sum_{x \in \chi} g(|x-\varphi(x)|)(\theta(x-z)+\theta(\varphi(x)-z)), \varphi: \chi \rightarrow \mathcal{L}^{b} \text { bijective }\right\},
$$

and, analogously, for $z \in \mathbb{R}_{w}^{3}$,

$$
e(\chi, z)=\inf \left\{\sum_{x \in \chi} g(|x-\varphi(x)|)(\theta(x-z)+\theta(\varphi(x)-z)), \varphi: \chi \rightarrow \mathcal{L}^{w} \text { bijective }\right\} .
$$

This approximate local energy density has a simple interpretation, cf. Fig. 3. Thus, suppose that $z \in \mathbb{R}_{b}^{3}$, the case $z \in \mathbb{R}_{w}^{3}$ following mutatis mutandi, and suppose that $|z \cdot \nu|>\lambda$, i. e., the distance of $z$ to the interface is greater than the radius of interaction. Then, $z$ sees atoms that are all on the same side of the interface, hence on $\mathcal{L}^{b}$, and the corresponding local energy density vanishes. Thus, as a function of $z$, the local energy density is restricted to a layer of thickness $2 \lambda$ around the plane $\Sigma$ of the interface. Suppose, contrariwise, that $z \in \mathbb{R}_{b}^{3}$, and $|z \cdot \nu|<\lambda$, i. e., the distance of $z$ to the interface is less than the radius of interaction. In this case, the atoms within $B(z, \lambda)$ on the same side of the interface are on $\mathcal{L}_{b}$, whereas the reminder of the atoms on the opposite side of the interface are on $\mathcal{L}_{w}$. The local energy density is then given by the cost of mapping the $\mathbb{R}_{w}^{3}$-atoms to those on $\mathcal{L}_{b}$.

A further simplification - and more explicit expressions - of the energy result from the use of the $L^{2}$ upper bound (15) as an approximation to the local energy. Thus, for $z \in \mathbb{R}_{b}^{3}$, inserting the definition of $\rho_{\beta}$ into (15) gives

$$
\begin{aligned}
e^{\beta}(\chi, z) & =\int_{B_{\lambda}(z)} c\left|\rho_{\beta}^{\chi}(p)-\rho_{\beta}^{\mathcal{L}^{b}}(p)\right|^{2} \theta(p-z) d p \\
& =\int_{B_{\lambda}(z)} c\left|\sum_{x \in \chi} \psi_{\beta}(x-p)-\sum_{y \in \mathcal{L}^{b}} \psi_{\beta}(y-p)\right|^{2} \theta(p-z) d p .
\end{aligned}
$$




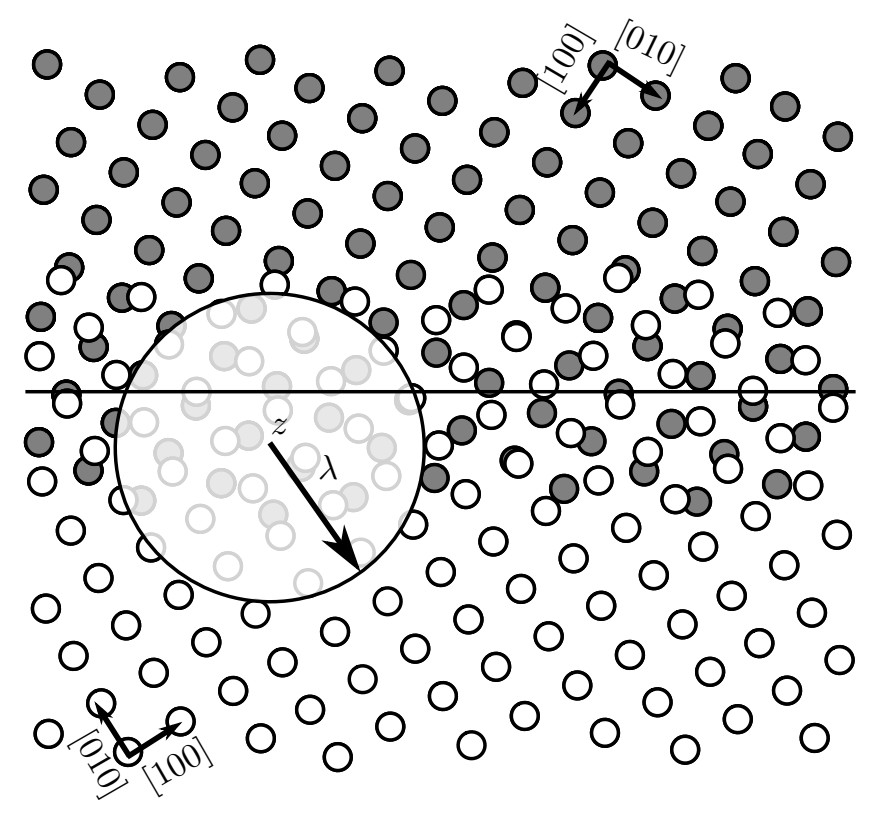

Figure 3: Rigid lattice model of interface between two misoriented half-lattices where the horizontal line is the virtual interface plane. The local interface energy (i.e. continuous intensive energy as a function of position $z$ ) is the local cost of deformation (i.e. within a radius $\lambda$ from $z$ ) of deformation of one lattice to the other.

Since $\chi$ coincides with $\mathcal{L}^{b}$ on $\mathbb{R}_{b}^{3}$, we equivalently have

$$
e^{\beta}(\chi, z)=\int_{B_{\lambda}(z)} c\left|\sum_{x \in \mathcal{L}^{w} \cap \mathbb{R}_{w}^{3}} \psi_{\beta}(x-p)-\sum_{y \in \mathcal{L}^{b} \cap \mathbb{R}_{w}^{3}} \psi_{\beta}(y-p)\right|^{2} \theta(p-z) d p .
$$

Since $z \in \mathbb{R}_{b}^{3}$, and $\theta$ and $\psi_{\beta}$ have compact support, the integral localizes to a strip around the interface. Again, as in the case of the rigid-lattice approximation, we note that the particular choice of local rotations and translations just described results in an upper bound of the energy.

In summary, the approximate interfacial energy per unit area that results from a rigid lattice approximation and the hard-wiring of the local rotations and translations according to position relative to the interface is

$$
\begin{aligned}
\gamma \approx \lim _{L \rightarrow \infty} \frac{1}{L^{2}}( & \int_{\Omega_{L, \nu} \cap \mathbb{R}_{b}^{3}}\left(\int_{B_{\lambda}(z)} c\left|\sum_{x \in \mathcal{L}^{w} \cap \mathbb{R}_{w}^{3}} \psi_{\beta}(x-p)-\sum_{y \in \mathcal{L}^{b} \cap \mathbb{R}_{w}^{3}} \psi_{\beta}(y-p)\right|^{2} \theta(z-p) d p\right) d z+ \\
& \left.\int_{\Omega_{L, \nu} \cap \mathbb{R}_{w}^{3}}\left(\int_{B_{\lambda}(z)} c\left|\sum_{x \in \mathcal{L}^{b} \cap \mathbb{R}_{b}^{3}} \psi_{\beta}(x-p)-\sum_{y \in \mathcal{L}^{w} \cap \mathbb{R}_{b}^{3}} \psi_{\beta}(y-p)\right|^{2} \theta(z-p) d p\right) d z\right) .
\end{aligned}
$$

Here $\Omega_{L, \nu}$ is a cube of side $L$ centered in the origin with one face normal to $\nu$. We note, in particular, that all integrals can be localized to a finite layer around the interface, as the integrand vanishes elsewhere.

The above result is an analytic expression for computing an upper bound on the grain boundary energy in its most general form. We reiterate that many phenomena are consequently excluded from the above result; specifically, microscopic mechanisms for relaxation as a result of misfit (c.f. [60, 69]) are not accounted for explicitly. This is in accordance with the model as one that finds the upper bound of the grain boundary energy; we expect accounting for microscopic relaxation mechanisms to reduce the energy. In the direct 
application of this model to homophase boundaries, we assume that energy of microscopic relaxation is negligible compared to the energy of perfect lattice mismatch.

\section{Results for Homophase Boundaries}

In the previous section we developed a general expression for computing interface energy. In this section we reduce and specialize the expression, resulting in a compact formula for energy that is amenable to direct computation.

The space of general interface orientations is conventionally thought of as a five-dimensional space, three degrees of freedom corresponding to the misorientation between the crystals and the remaining two degrees of freedom corresponding to the unit normal. This space is too unwieldy for general exploration. Consequently, we restrict the scope of the validation tests to a subset of well-understood interface classes, with the expectation that consistent behavior for these interface classes is an indicator of the predictive nature of the model. The choice of these interface classes is entirely driven by the availability of validation data. The data for interface energy, in lieu of direct experimental measurement, is taken from molecular dynamics (MD) simulations. In this vein, we consider homophase interfaces or grain boundaries (GBs), that is, interfaces at which a rotational transformation exists that transforms one crystal lattice to the other. We consider the following three types of interfaces: symmetric tilt grain boundaries (STGB), symmetric twist grain boundaries (STwGB) and asymmetric twist grain boundaries (ATwGB). Additionally, we consider materials whose lattice structure falls into one of three classes: face-centered cubic (FCC), body-centered cubic (BCC) and hexagonal close-packed (HCP). Two or more materials are considered for each choice of crystal structure and boundary class, with the choice of materials driven by the availability of MD data.

\subsection{Evaluation of the energy}

All comparisons with experiment are based on the upper-bound approximation (24). In order to simplify the calculations, we expand the square in the bound (15), as well as in all subsequently derived expressions thereof, as

$$
\begin{aligned}
& \int_{\mathbb{R}^{3}}\left|\rho_{\beta}^{X}(x)-\rho_{\beta}^{Y}(x)\right|^{2} \theta(x-z) d x= \\
& \int_{\mathbb{R}^{3}}\left|\rho_{\beta}^{X}(x)\right|^{2} \theta(x-z) d x+\int_{\mathbb{R}^{3}}\left|\rho_{\beta}^{Y}(x)\right|^{2} \theta(x-z) d x-2 \int_{\mathbb{R}^{3}} \rho_{\beta}^{X}(x) \rho_{\beta}^{Y}(x) \theta(x-z) d x .
\end{aligned}
$$

When integrated over the domain of the crystal, the last term on the right-hand side gives the covariance of the densities $\rho_{\beta}^{X}$ and $\rho_{\beta}^{Y}$, whereas the other two terms ensure that the energy is positive and vanishes for $\rho_{\beta}^{X}=\rho_{\beta}^{Y}$. This expansion affords the additional inside that interfacial energy is controlled by the covariance between the atomic positions in the vicinity of the interface. In calculations, we evaluate covariances numerically, with the remaining two terms in (25) estimated as the requisite offsets that render the interfacial energy positive.

The three-dimensional integral over $\Omega_{L, \nu}$ in the calculation of the covariance may be further decomposed into an integral within the plane $\Sigma$ of the interface and an integral in the normal direction $z \cdot \nu$. The $L^{2}$-product structure of the covariance conveniently lends itself to an efficient evaluation of the in-plane integral using Fourier series. Likewise, the areal density limit of $L \rightarrow+\infty$ is also conveniently performed in Fourier space through an Abel regularization of the energy sum [70]. In calculations, $L$ ranges between 10 and 20 lattice spacings. We note that many high-angle boundaries have much greater repeating units, and the repeating unit incommensurate boundaries is infinite. However in practice we see that the choice of $L$ is in general irrelevant for boundaries with large repeating units as their energy is already very high.

The transverse integral in the normal direction may be restricted to $[-\lambda, \lambda]$, with $\lambda$ the range of interaction or, equivalently, the radius of the support of the localization function $\theta$. This integral can be performed by Gaussian quadrature. In calculations, we use the simplest possible Gaussian-quadrature rule consisting of one single point. This choice effectively restricts the three-dimensional integral over $\Omega_{L, \nu}$ to a twodimensional integral over $\Sigma$. When considering the relaxation of the material it must be considered that 


\begin{tabular}{ccccc}
\hline & $\mathrm{Cu}(\mathrm{FCC})$ & $\mathrm{Au}(\mathrm{FCC})$ & $\mathrm{Mo}(\mathrm{BCC})$ & $\mathrm{Fe}(\mathrm{BCC})$ \\
\hline$c\left(J / m^{2}\right)$ & 1.0 .95 & 2.525 & 1.950 & \\
$\sigma / a$ & 0.175 & 0.175 & 0.19 & 0.19 \\
\hline
\end{tabular}

Table 2: Material parameter values used in all GB tests. $\sigma / \alpha$ is the nondimensional temperature (dependent on the temperature, the atomic embedding energy, and the lattice constant) and $c$ is a linear scaling constant. Values are currently determined by curve fitting.

the effects of relaxation may be present for a considerable distance in the transverse direction. However, as this treatment considers idealized lattices with no relaxation, a reduced integral suffices. We also note that the formulation does not preclude the use of a reduced integration scheme; nevertheless for this application such an extension is unnecessary.

We choose the thermalization mollifier to be a Gaussian function of the form

$$
\psi_{\beta}(x)=\frac{1}{Z} \mathrm{e}^{-\beta(m / 2) \omega^{2}|x|^{2}},
$$

with

$$
Z=\left(\frac{2 \pi}{\beta m \omega^{2}}\right)^{3 / 2}
$$

which may be regarded as a mean-field approximation of the Gibbs canonical distribution (cf., e. g., [71]). Finally, the shift between the abutting lattices is fixed simply by making them have one common atom. These choices and approximations render the evaluation of the energy essentially explicit.

The length parameter $\sigma=1 / \sqrt{\beta(m / 2) \omega^{2}}$ and the energy constant $c$ in the bound (24) are the sole free parameters of the model and are determined by fitting to the experimental data. The MD data chosen for comparison in this work is taken from a series of papers by Wolf [14-24], which provide comprehensive data from a wide range of interface configurations using a consistent methodology. The results of the fitting are tabulated in Table 2, with $\sigma$ normalized by the lattice parameter $a$.

\subsection{Grain Boundary Geometry}

Three types of grain boundary geometries are considered in this section.

Symmetric tilt grain boundaries, cf. Fig. 4a, are obtained by rotating two halves of a uniform crystal symmetrically with respect to a prescribed interface. Thus all STGBs are defined by i) the crystallographic direction (in Miller indices) corresponding to the tilt axis that remains constant, ii) the total tilt angle between the two crystals about the tilt axis, and iii) the direction corresponding to the initial normal axis to the homogeneous interface. Because STGBs are by definition symmetric about the constant interface plane, the tilt axes must lie within a symmetry plane of the crystal [31]; as such the class of STGBs is limited to a selection of tilt axes. For cubic materials the STGB tilt axes are $\langle 100\rangle,\langle 110\rangle,\langle 111\rangle$, and $\langle 112\rangle$, and we have found a wide selection of MD data for these STGBs [14-16, 22-24].

Symmetric twist grain boundaries, cf. Fig. 4c, are obtained by an antisymmetric rotation of upper and lower halves of the crystal about a common axis (the "twist axis") that is normal to the initial interface plane. The twist axis and twist angle are sufficient to characterize any STwGB grain boundary. As with STGBs, the class of STwGBs are restricted to those associated with twist axes for which the corresponding normal is a symmetry plane of the material. Asymmetric twist grain boundaries are defined similarly to STwGBs, but without the restriction of a common twist axis between the materials (thereby eliminating any restrictions on the symmetry of the interface). Classes of ATwGBs are specified using the two crystallographic directions corresponding to the normal vectors of the top and bottom crystals.

\subsection{FCC symmetric tilt grain boundaries}

The first set considered are the FCC symmetric tilt grain boundaries. The results of the comparison for the $[100],[110],[111]$ and [112] boundaries are presented in Fig. 5. We compare and fit the model results 


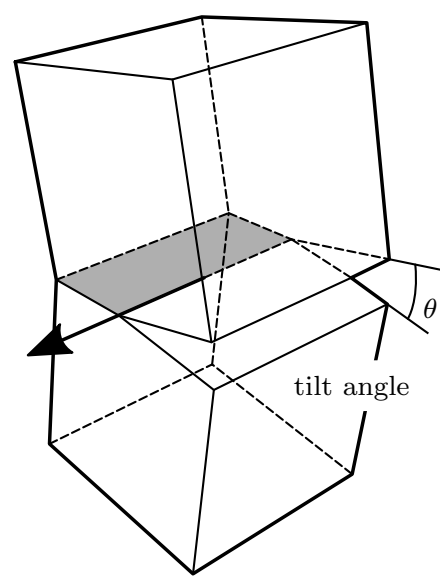

(a) STGB

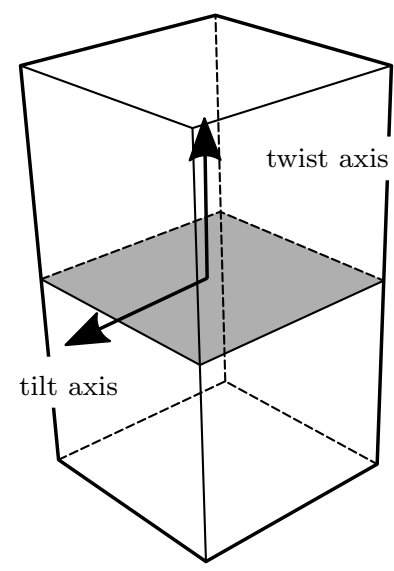

(b) Homogeneous

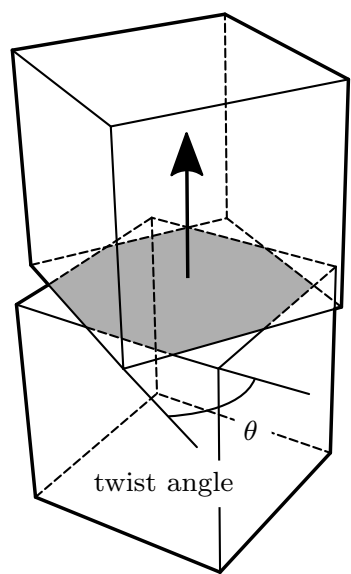

(c) TwGB

Figure 4: We begin with a uniform crystal with no boundary (center). A symmetric tilt grain boundary is formed by symmetric rotation about an in-plane tilt axis (left). A symmetric twist grain boundary results from asymmetric rotation about the normal twist axis (right).

to MD results from [14] for gold, using an embedded atom method (EAM) potential, and copper, using both EAM and Lennard-Jones (LJ) potentials. We have computed the grain boundary energy using the covariance model (the hashed line), and observed a close match of the model to the MD results.

For the [100] tilt boundaries, the cusp corresponding to the $\{110\}$ boundary is observed at $\theta=90^{\circ}$. For the [110] tilt boundaries, we observe the cusps corresponding to the $\{111\}$ and $\{113\}$ boundaries at $\theta=70.6^{\circ}$ and $\theta=129.4^{\circ}$, respectively. For the [111] tilt boundaries, other than the cusp corresponding to the homogeneous case at $\theta=0^{\circ}$, we do not observe any significant energy cusps. For the [112] tilt boundaries, we observe a deep cusp corresponding to the $\{113\}$ boundary. The location of all of the deep energy cusps for $\theta>0$ predicted by the MD data are matched exactly, to varying accuracy, by the model.

We observe a discrepancy between the model and the MD data at the $\{100\}$ homogeneous case $(\langle 100\rangle$ at $\left.\theta=0^{\circ}\right)$ and the $\{110\}$ homogeneous case $\left(\langle 110\rangle,\langle 111\rangle,\langle 112\rangle\right.$ STGB at $\left.\theta=0^{\circ}\right)$. Although the location of the cusps corresponding to the homogeneous case is correct, we observe that the model overpredicts the energy. Addressing this issue requires additional considerations of relaxation which can be captured by MD. This issue will be discussed subsequently.

\subsection{BCC symmetric tilt grain boundaries}

The next set of boundaries considered are the symmetric tilt grain boundaries for BCC crystals, with the same configurations and $\theta$ ranges as for FCC STGBs. We compare the results of the model with MD results from [22] for $\alpha$-Iron (Ferrite) using a Johnson potential, and Molybdenum using a Finnis-Sinclair type potential. The model results are plotted in Figure 6. We observe close agreement between the predictions of the model and with the MD results.

For the $[100]$ tilt, we observe strong energy cusps at $\theta=0^{\circ}, 90^{\circ}$, corresponding to the $\{100\}$ and $\{110\}$ STGBs. A weak cusp is also present at $\theta=37^{\circ}$ corresponding to the $\{310\}$ STGB. For [110] tilt, we observe strong energy cusps at $\theta=0,109^{\circ}, 180^{\circ}$, corresponding to the $\{110\},\{112\},\{100\}$ boundaries. We note that the cusp at $\theta=51^{\circ}$ corresponding to the $\{332\}$ STGB is only barely noticeable in the model, whereas it is a significant cusp in the MD data. On the other hand, the model predicts a cusp at $\theta=70^{\circ}$ corresponding to the $\{111\}$ STGB that is not present in the MD data. We refer the reader to alternative MD simulations (e.g. [72]) of the same boundaries that also do not predict the $\{332\}$ cusp. For the [111] tilt boundaries we observe deep cusps at $\theta=0^{\circ}$ and $\theta=90^{\circ}$, corresponding to the $\{100\}$ and $\{110\}$ STGBs. A minor cusp is observed at $\theta=38^{\circ}$ corresponding to the $\{231\}$ STGBs. 

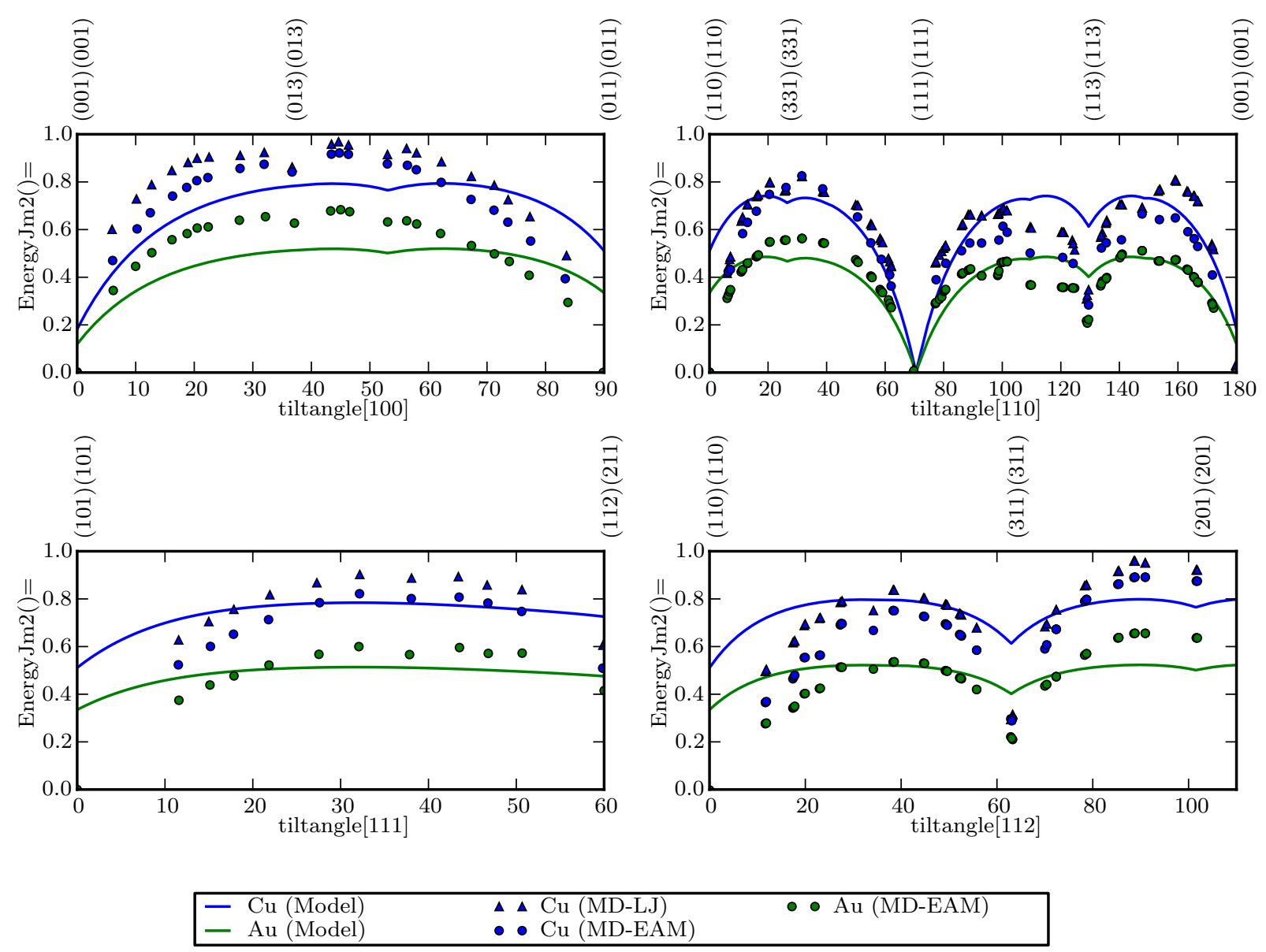

Figure 5: FCC STGB prediction of the covariance model for Copper and Gold, compared with molecular dynamics results using the Embedded Atom Method (EAM) and Lennard-Jones (LJ) potentials. MD results from [14]. Annotations on the top indicate the boundary plane for the top crystal at cusp locations.

As with the FCC STGBs, we observe that the magnitude of the cusps-in particular, the cusps corresponding to homogeneous cases-do not appear to agree with the MD data. Likely sources of these discrepanciesand possible remedies thereof-are discussed in Section 4.

\subsection{FCC twist grain boundaries}

The next set of boundaries considered are the FCC twist grain boundaries. There are two types of these boundaries: asymmetric twist (ATwGB) and symmetric twist (STwGB). The STwGBs are created by beginning with a homogeneous crystal, defining a twist axis, and rotating the top and bottom crystals symmetrically about it. ATwGBs are a more general class of boundaries, and are defined by the two grain boundary planes of the top and bottom crystals.

The results for the STwGBs are, in general, as expected, with deep cusps corresponding to the homogeneous cases at $\theta=0,90^{\circ}$ and $\theta=0,60^{\circ}$. Results are compared to MD work by Wolf [16] in the first two plots in Figure 7. However we see a greater difference for the STwGBs than any other of the FCC tests; the $\{111\}$ STwGB showing a particularly high value above the MD results. It appears that the results differ only by a multiplicative constant and we note that the model results could easily be re-scaled for a better fit. However, as the the same set of material parameters are used for all GB calculations, it would not be possible to adjust $E_{0}$ without significantly altering the fit to other data. We suggest as possible explanations 

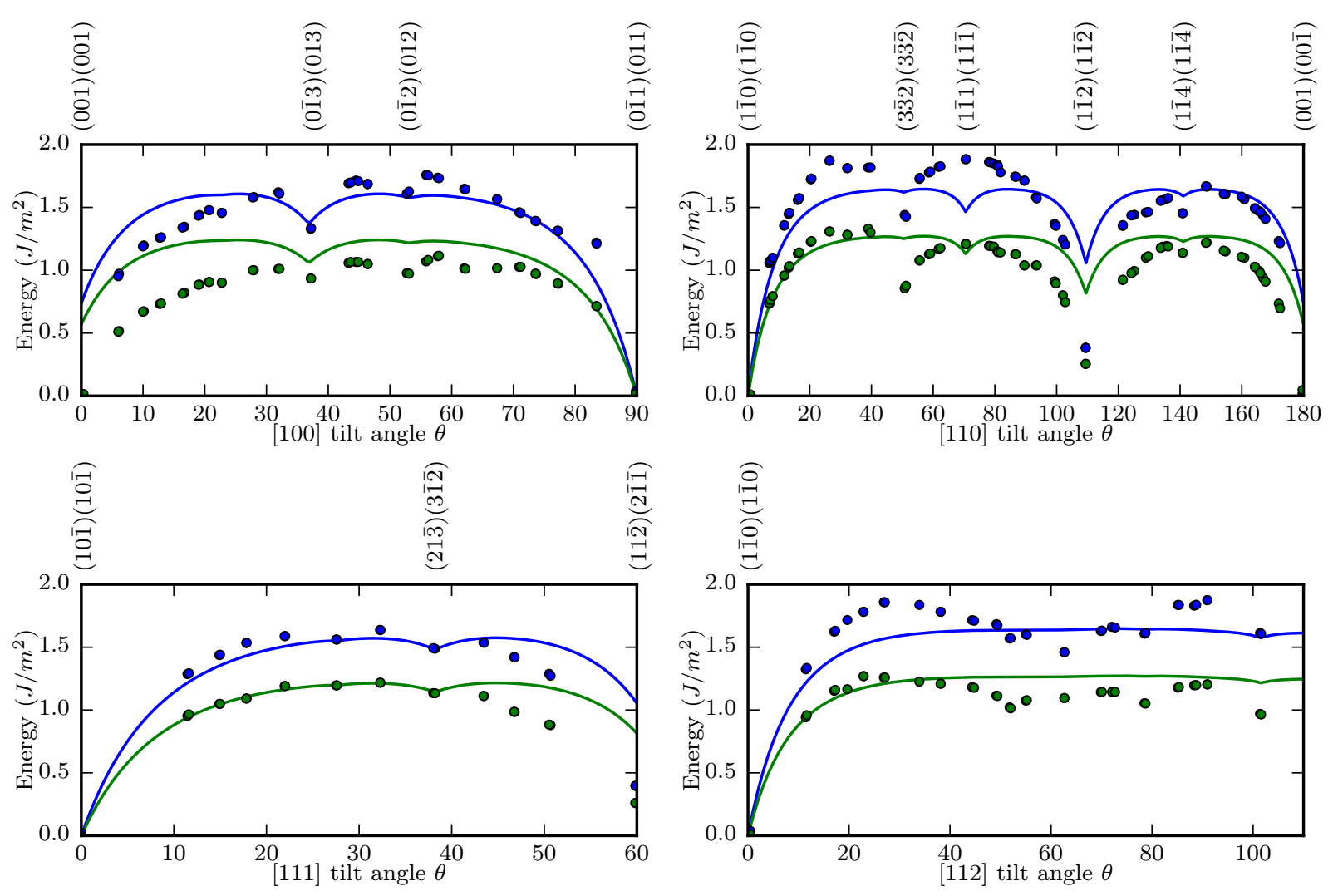

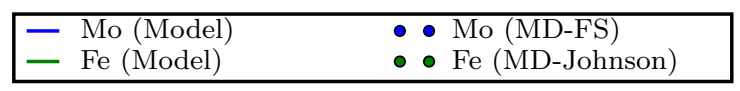

Figure 6: BCC STGB results of the covariance model for Molybdenum and Iron, compared with MD results using FS (for Mo) and Johnson (for Fe) potentials. MD results from [22]. Annotations on the top indicate the boundary plane for the top crystal at cusp locations.

that: i) the MD results attain a relaxation state that is inaccessible by the model, or ii) variations in MD results due to use of different interatomic potentials.

Notably, the results for the asymmetric twist boundaries are more accurate. The results are again compared to MD work by Wolf [16] in the last four plots in Figure 7. We notice almost exact agreement for the (115)(111) and (221)(001) boundaries, and a fairly close estimate of the energy for the (114)(011) and (557)(113) boundaries. In addition, we note that the cusp location is predicted exactly as well. The cusp magnitude for some of the cases, particularly (114)(011) and (221)(001), differ significantly.

\subsection{BCC twist grain boundaries}

The final set of grain boundaries investigated were BCC STwGB and ATwGB configurations. The selection of the investigated boundaries were driven by the available MD data.

The results for the model for STwGB boundaries are compared against MD data from Wolf $[23,24]$ in Figure 8. For the $\{001\}$ STwGB we notice close agreement with the MD data, matching the minor cusps at $\theta=37^{\circ}, 53^{\circ}$. The results for $\{011\}$ are surprisingly similar to the MD results, differing only by a multiplicative factor. For the $\{031\},\{111\}$ and $\{112\}$ boundaries, the MD data is somewhat chaotic and we do not observe a significant trend in the data or the presence of cusps. Further, we notice that the symmetry present in these lattices does not appear to be present in the MD data. Finally we note that, as with previous examples, the absolute values of many of the deep energy cusps differs from that predicted by the model. 

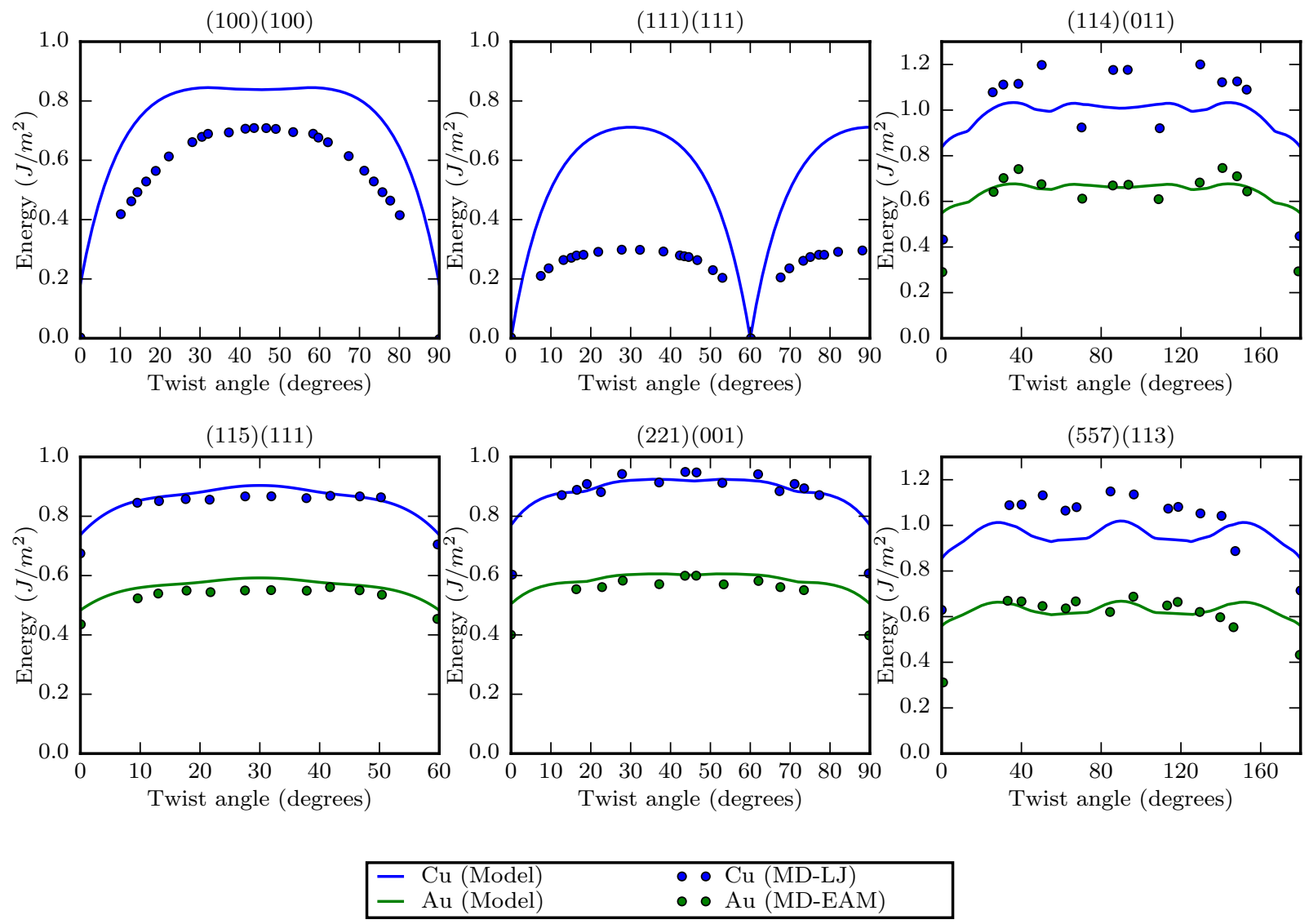

Figure 7: FCC TwGB prediction of the covariance model for Copper and Gold, compared with MD results using EAM (for gold) and LJ (for copper). MD results for symmetric twist from [15], asymmetric twist from $[16]$.

Finally, the results for BCC ATwGBs are presented with MD data from Wolf [24] in Figure 9. For the (114)(011) we notice an exceedingly close match, with all of the cusps and magnitudes almost identical. For the other families of ATwGBs we notice a somewhat chaotic tendency in the MD data without many distinguishing features. One exception is the data for Fe in the (221)(001) configuration-we observe apparent cusps at $\sim 37^{\circ}$ and $\sim 53^{\circ}$ that is not captured by the data. However, the cusps are clearly not present in the Mo case, an apparent example of material dependence in the behavior of the energy landscape.

\subsection{Results summary}

We find that we obtain reasonable qualitative agreement between the model and the molecular dynamics data. In particular, the precise location of all significant energy cusps are recovered. Additionally the model recovers the general shape of the energy function with reasonable fidelity. Table 3 itemizes each test case along with its RMS error and list of predicted cusp locations. In general we observe recovery by the model of the MD data with less than $25 \%$ error, and most results ranging from $10 \%-20 \%$. Significant outliers include, e.g. FCC (111)(111) twist and BCC (011)(011), causing a very high RMS error percentage as a result of the linear scaling difference between the model and MD data. Nevertheless we find the average error to be acceptable given the consistency of scaling parameters across all of the data sets. 
(001)(001)

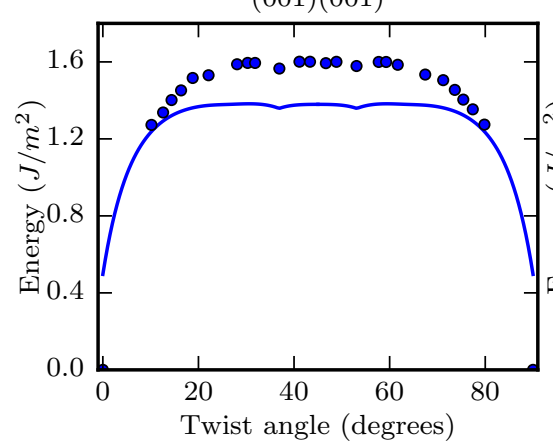

(111)(111)

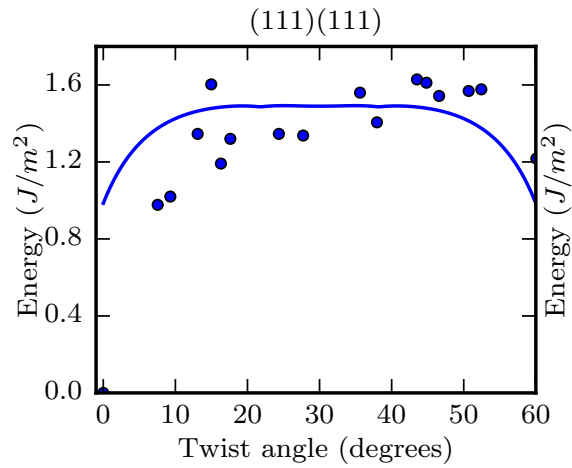

(011)(011)

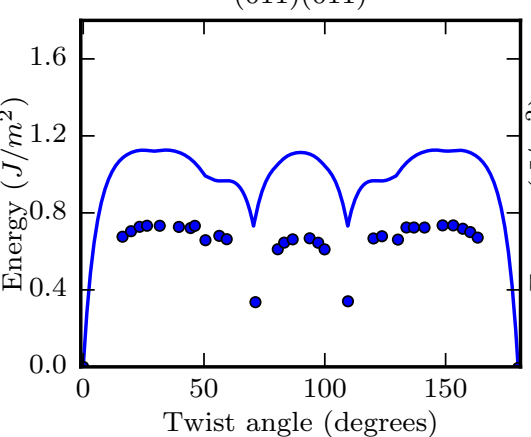

(112)(112)

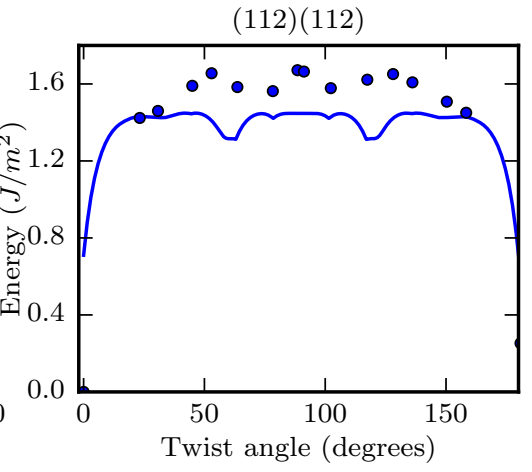

(031)(031)

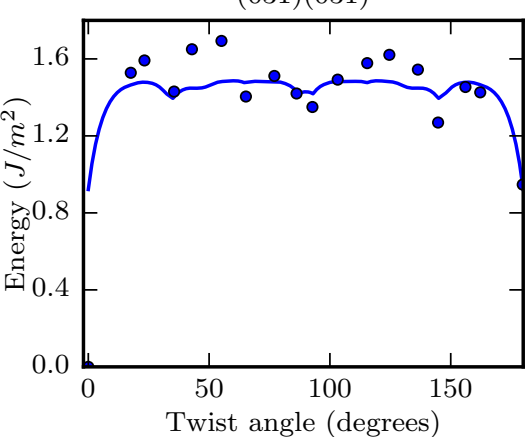

- Fe (Model)

- - Fe (MD-Johnson)

Figure 8: BCC STwGB. MD data from [23, 24]
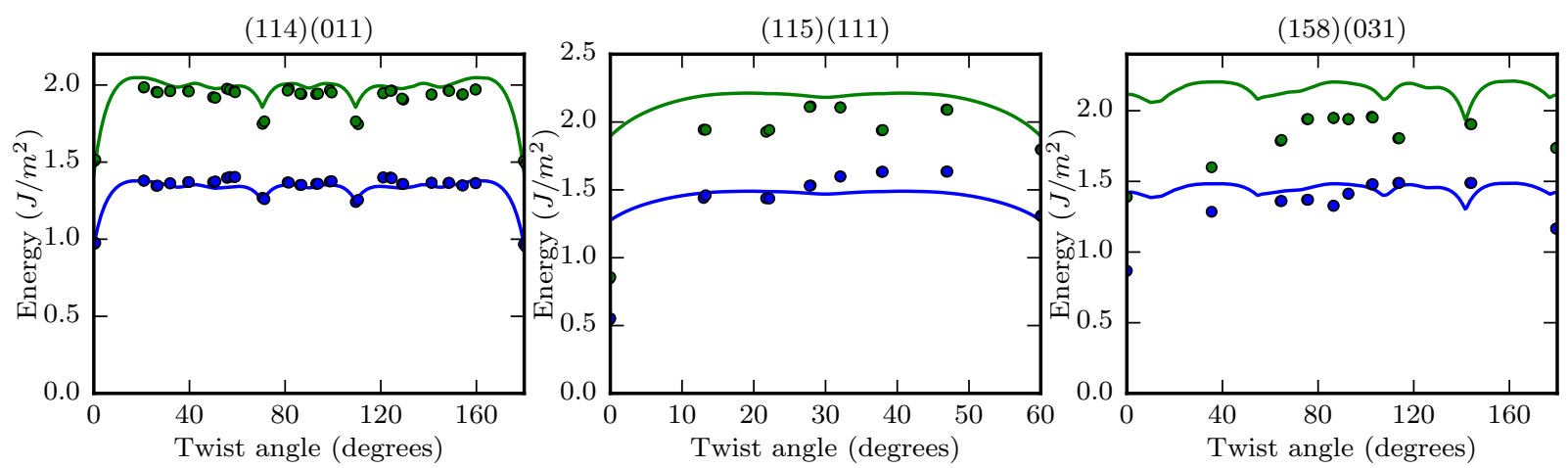

(221)(001)
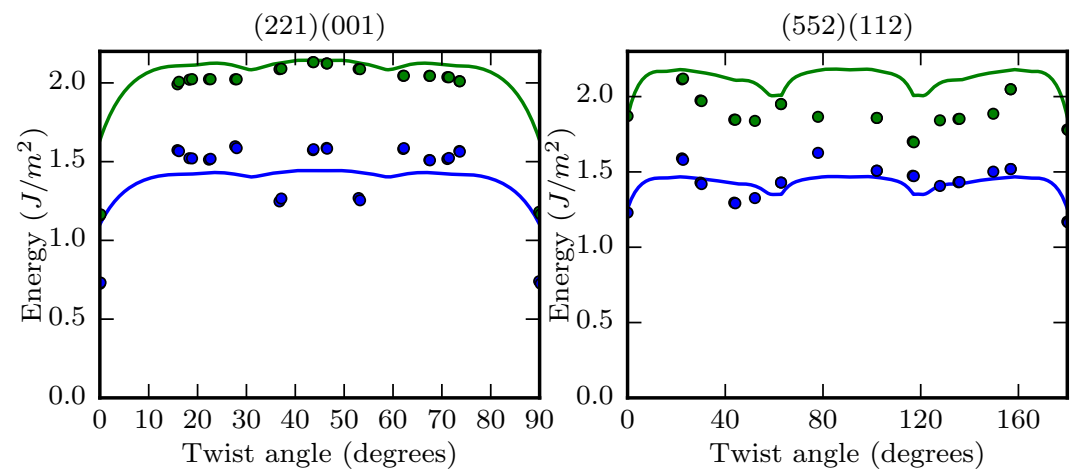

- Mo (Model)

- Fe (Model)

- Mo (MD-FS)

- $\mathrm{Fe}$ (MD-Johnson)

Figure 9: BCC ATwGB. MD data from [24] 


\section{Concluding remarks}

We have developed a new class of lattice matching interatomic energies that build into their definition information about the stable phases of the crystal in a reference-free manner. In essence, lattice matching interatomic energies accord a cost to transporting local clusters of atoms to a locally matched perfect-lattice configuration. They also lend themselves to analysis and, in particular, to estimation by functional-analytical means. We use one such estimate to simplify and streamline the evaluation of interfacial energies, which is rendered essentially analytical. Despite the small number of free parameters in the model (two) and despite the approximations adopted in calculations, the model predicts cusp locations, captures trends in the energy, and approximates large-angle boundary energy data. Because all results are obtained using consistent material parameters and no a priori information regarding cusp location or magnitude, we find these findings to provide validation of the predictive ability of the model over a wide range of grain boundary geometries.

These encouraging results suggest multiple avenues for further research. Out of all possible interface orientations, only a small subset has been explored. Other orientations, such as asymmetric tilt grain boundaries, other crystal structures, such as hexagonal close-packed, and multiphase/multi-material boundaries, such as FCC-BCC Kurdjumov-Sachs are well-within the scope of the model. The model has also immediate applications to the study of mesoscopic phenomena, such as recrystallization, solidification, severe plastic deformation, and others. The integration of the model into multi-scale mesoscopic models of plasticity and its implementation into large-scale computational frameworks raises additional challenges and opportunities.

We close by acknowledging some shortcomings of the model. Some of the discrepancies with MD data may undoubtedly be attributed to uncertainty in the MD empirical potentials, resulting in large discrepancies between MD sets themselves [24]. However the drastic simplification of grain boundaries to a 5 degreeof-freedom description necessitates a number of simplifications. To sufficiently reduce the order of the model, the degrees of freedom necessary for modeling complex relaxation configurations at a grain boundary or interface are not considered; each deformed lattice is matched to its reference lattice. An additional approximation that is made in the model is the assumption that the interface is planar; in reality, many interfaces relax their energy through the development of faceted microstructures [64]. Faceting constructions taking the present interfacial model as input thus suggest themselves as a means of further relaxing the interfacial energy and, in the process, closing some of the gaps between the unrelaxed model and the data. However, this extension of the theory is beyond the scope of the present paper and will be pursued in a separate publication. We remark that the positions of the cusps determine the preferred orientations of the facets. Since the current model is very accurate in the prediction of the cusps, we expect that the resulting facet structures will also be accurately predicted. Finally, the model accounts for lattice thermalization, however the assumption is made that thermal fluctuations are decoupled; it is expected that this may be a cause of error especially for high temperatures.

\section{Acknowledgments}

Brandon Runnels and Michael Ortiz would like to thank the NNSA's High Energy Density Laboratory Plasmas program under award \#DE-NA0001805. Brandon Runnels additionally thanks the Los Alamos National Laboratory Seaborg Institute for support during Summer 2014. Irene Beyerlein would like to acknowledge support by a Laboratory Directed Research and Development program award number 20140348ER. Sergio Conti would like to acknowledge support of the DFG unter SFB 1060, project A6.

\section{References}

[1] R. Doherty, D. Hughes, F. Humphreys, J. Jonas, D. Jensen, M. Kassner, W. King, T. McNelley, H. McQueen, and A. Rollett, "Current issues in recrystallization: a review," Materials Science and Engineering: A, vol. 238, pp. 219-274, Nov. 1997.

[2] T. Watanabe and S. Tsurekawa, "The control of brittleness and development of desirable mechanical properties in polycrystalline systems by grain boundary engineering," Acta Materialia, vol. 47, pp. 4171-4185, Nov. 1999. 
[3] A. Demirel, MC and Kuprat, AP and George, DC and Rollett, "Bridging Simulations and Experiments in Microstructure Evolution," Physical Review Letters, vol. 90, p. 016106, Jan. 2003.

[4] S. Zheng, I. J. Beyerlein, J. S. Carpenter, K. Kang, J. Wang, W. Han, and N. A. Mara, "High-strength and thermally stable bulk nanolayered composites due to twin-induced interfaces.," Nature communications, vol. 4, p. 1696, Jan. 2013.

[5] I. J. Beyerlein, J. R. Mayeur, S. Zheng, N. A. Mara, J. Wang, and A. Misra, "Emergence of stable interfaces under extreme plastic deformation.," Proceedings of the National Academy of Sciences of the United States of America, vol. 111, pp. 4386-4390, Mar. 2014.

[6] C. Herring, "Structure and properties of solid surfaces," Journal of Chemical Education, p. 5, 1954.

[7] C. Herring, The physics of powder metallurgy. McGraw-Hill, 1951.

[8] N. Gjostein and F. Rhines, "Absolute interfacial energies of [001] tilt and twist grain boundaries in copper," Acta Metallurgica, vol. 7, pp. 319-330, May 1959.

[9] A. Wright and S. Atlas, "Density-functional calculations for grain boundaries in aluminum," Physical Review B, vol. 50, pp. 15248-15260, Nov. 1994.

[10] J. Hartford, "Interface energy and electron structure for Fe/VN," Physical Review B, vol. 61, pp. 2221-2229, Jan. 2000.

[11] P. Gehlen, J. Hirth, R. Hoagland, and M. Kanninen, "A new representation of the strain field associated with the cube-edge dislocation in a model of a $\alpha$-iron," Journal of Applied Physics, vol. 43, no. 10, pp. 3921-3933, 1972.

[12] K. Kang, J. Wang, S. J. Zheng, and I. J. Beyerlein, "Minimum energy structures of faceted, incoherent interfaces," Journal of Applied Physics, vol. 112, no. 7, p. 73501, 2012

[13] M. Daw and M. Baskes, "Embedded-atom method: Derivation and application to impurities, surfaces, and other defects in metals," Physical Review B, 1984.

[14] D. Wolf, "Structure-energy correlation for grain boundaries in F.C.C. metalsIII. Symmetrical tilt boundaries," Acta Metallurgica et Materialia, vol. 38, pp. 781-790, May 1990.

[15] D. Wolf, "Structure-energy correlation for grain boundaries in F.C.C. metals I: Boundaries on the (111) and (100) planes," Acta Metallurgica, vol. 37, pp. 1983-1993, July 1989.

[16] D. Wolf, "Structure-energy correlation for grain boundaries in f.c.c. metals IV: Asymmetrical twist (general) boundaries," Acta Metallurgica et Materialia, vol. 38, pp. 791-798, May 1990.

[17] C. Schmidt, M. W. Finnis, F. Ernst, and V. Vitek, "Theoretical and experimental investigations of structures and energies of $\Sigma=3$, [112] tilt grain boundaries in copper," Philosophical Magazine A, vol. 77, pp. 1161-1184, May 1998.

[18] U. Wolf, F. Ernst, T. Muschik, M. W. Finnis, and H. F. Fischmeister, "The influence of grain boundary inclination on the structure and energy of $\sigma=3$ grain boundaries in copper," Philosophical Magazine A, vol. 66, pp. 991-1016, Dec. 1992.

[19] K. L. Merkle and D. Wolf, "Low-energy configurations of symmetric and asymmetric tilt grain boundaries," Philosophical Magazine A, vol. 65, pp. 513-530, Feb. 1992.

[20] M. A. Tschopp and D. L. McDowell, "Structures and energies of $\Sigma 3$ asymmetric tilt grain boundaries in copper and aluminium," Philosophical Magazine, vol. 87, pp. 3147-3173, Aug. 2007.

[21] M. A. Tschopp and D. L. Mcdowell, "Asymmetric tilt grain boundary structure and energy in copper and aluminium," Philosophical Magazine, vol. 87, pp. 3871-3892, Sept. 2007.

[22] D. Wolf, "Correlation between the energy and structure of grain boundaries in b.c.c. metals. II. Symmetrical tilt boundaries," Philosophical Magazine A, vol. 62, pp. 447-464, Oct. 1990.

[23] D. Wolf, "Correlation between the energy and structure of grain boundaries in b.c.c. metals I. Symmetrical boundaries on the (110) and (100) planes," Philosophical Magazine Part B, vol. 59, pp. 667-680, June 1989.

[24] D. Wolf, "Structure and energy of general grain boundaries in bcc metals," Journal of Applied Physics, vol. 69, p. 185, Jan. 1991.

[25] J. Wang and I. J. Beyerlein, "Atomic structures of symmetric tilt grain boundaries in hexagonal close packed (hcp) crystals," Modelling and Simulation in Materials Science and Engineering, vol. 20, p. 024002, Mar. 2012.

[26] J. Wang and I. J. Beyerlein, "Atomic Structures of [0-110] Symmetric Tilt Grain Boundaries in Hexagonal Close-Packed (hcp) Crystals," Metallurgical and Materials Transactions A, vol. 43, pp. 3556-3569, May 2012.

[27] K. Kang, J. Wang, and I. J. Beyerlein, "Atomic structure variations of mechanically stable fcc-bcc interfaces," Journal of Applied Physics, vol. 111, no. 5, p. 53531, 2012.

[28] G. I. Taylor, "The mechanism of plastic deformation of crystals. Part II. Comparison with observations," Proceedings of the Royal Society of London. Series A, Containing Papers of a Mathematical and Physical Character, pp. 388-404, 1934.

[29] W. Read and W. Shockley, "Dislocation Models of Crystal Grain Boundaries," Physical Review, vol. 78, 1950.

[30] D. Wolf, "A read-shockley model for high-angle grain boundaries," Scripta Metallurgica, vol. 23, pp. 1713-1718, Oct. 1989.

[31] A. P. Sutton and R. Balluffi, Interfaces in crystalline materials. Clarendon Press, 1995.

[32] J. H. van der Merwe, "On the Stresses and Energies associated with Inter-Crystalline Boundaries," Proceedings of the Physical Society. Section A, vol. 63, pp. 616-637, June 1950.

33] G. Schoeck, "The generalized PeierlsNabarro model," Philosophical Magazine A, vol. 69, pp. 1085-1095, June 1994.

[34] J. H. Van Der Merwe, "Crystal Interfaces. Part I. Semi-Infinite Crystals," Journal of Applied Physics, vol. 34, p. 117, Jan. 1963.

[35] R. Bullough, V. Tewary, and F. Nabarro, "Dislocations in Solids," FRN- Nabarro North-Holland, Amsterdam, 1979.

[36] F. Frank, "The resultant content of dislocations in an arbitrary intercrystalline boundary," Symposium on The Plastic Deformation of Crystalline..., 1950.

[37] B. A. Bilby, "Report of the Conference on Defects in Crystalline Solids," Tech. Rep. 12, Physical Society, Dec. 1954.

[38] P. van Beers, V. Kouznetsova, M. Geers, M. Tschopp, and D. McDowell, "A multiscale model of grain boundary structure and energy: From atomistics to a continuum description," Acta Materialia, vol. 82, pp. 513-529, Jan. 2015. 
[39] E. A. Holm, D. L. Olmsted, and S. M. Foiles, "Comparing grain boundary energies in face-centered cubic metals: Al, Au, $\mathrm{Cu}$ and Ni," Scripta Materialia, vol. 63, pp. 905-908, Nov. 2010.

[40] D. Brandon, B. Ralph, S. Ranganathan, and M. Wald, "A field ion microscope study of atomic configuration at grain boundaries," Acta Metallurgica, vol. 12, pp. 813-821, July 1964.

[41] D. Brandon, "The structure of high-angle grain boundaries," Acta Metallurgica, vol. 14, pp. 1479-1484, Nov. 1966.

[42] P. Goodhew and D. Smith, "Can the CSL model fail?," Scripta Metallurgica, vol. 14, pp. 59-61, Jan. 1980.

[43] V. Randle, "The coincidence site lattice and the sigma enigma," Materials Characterization, vol. 47, pp. 411-416, Dec. 2001.

[44] G. Bishop and B. Chalmers, "A coincidence Ledge Dislocation description of grain boundaries," Scripta Metallurgica, vol. 2, pp. 133-139, Feb. 1968.

[45] G. H. Bishop and B. Chalmers, "Dislocation structure and contrast in high angle grain boundaries," Philosophical Magazine, vol. 24, pp. 515-526, Sept. 1971.

[46] M. Weins, B. Chalmers, H. Gleiter, and M. Ashby, "Structure of high angle grain boundaries," Scripta Metallurgica, vol. 3, pp. 601-603, Aug. 1969.

[47] M. Weins, H. Gleiter, and B. Chalmers, "Structure of symmetric tilt boundaries in F. C. C. metals," Scripta Metallurgica, vol. 4, pp. 235-238, Mar. 1970.

[48] M. J. Weins, "Computer Calculations of the Structure and Energy of High-Angle Grain Boundaries," Journal of Applied Physics, vol. 42, p. 2639, Dec. 1971.

[49] W. Bollmann, Crystal defects and crystalline interfaces. Springer-Verlag, 1970.

[50] A. J. Vattré and M. J. Demkowicz, "Determining the Burgers vectors and elastic strain energies of interface dislocation arrays using anisotropic elasticity theory," Acta Materialia, 2013.

[51] J. Li, "Disclination model of high angle grain boundaries," Surface Science, vol. 31, pp. 12-26, June 1972.

[52] K. K. Shih and J. Li, "Energy of grain boundaries between cusp misorientations," Surface Science, vol. 50, no. 1, pp. 109$124,1975$.

[53] A. Nazarov, O. Shenderova, and D. Brenner, "On the disclination-structural unit model of grain boundaries," Materials Science and Engineering: A, vol. 281, pp. 148-155, Apr. 2000.

[54] C. Fressengeas, V. Taupin, and L. Capolungo, "Continuous modeling of the structure of symmetric tilt boundaries," International Journal of Solids and Structures, vol. 51, pp. 1434-1441, Mar. 2014.

[55] V. V. Bulatov, B. W. Reed, and M. Kumar, "Grain boundary energy function for fcc metals," Acta Materialia, vol. 65, pp. 161-175, Feb. 2014.

[56] S. Luckhaus and L. Mugnai, "On a mesoscopic many-body Hamiltonian describing elastic shears and dislocations," Continuum Mechanics And Thermodynamics, vol. 22, pp. 251-290, 2010.

[57] S. Luckhaus and J. Wohlgemuth, "Study of a model for reference-free plasticity," preprint arXiv.org 1408.1355, Aug. 2014.

[58] R. Johnson, "Relationship between two-body interatomic potentials in a lattice model and elastic constants," Physical Review B, vol. 6, no. 6, p. 2094, 1972 .

[59] V. Marichev, "The shuttleworth equation: Its modifications and current state," Protection of Metals and Physical Chemistry of Surfaces, vol. 47, no. 1, pp. 25-30, 2011.

[60] R. Dingreville, A. Hallil, and S. Berbenni, "From coherent to incoherent mismatched interfaces: A generalized continuum formulation of surface stresses," Journal of the Mechanics and Physics of Solids, vol. 72, pp. 40-60, 2014.

[61] J. Gibbs, H. Bumstead, and W. Longley, The collected works of J. Willard Gibbs. Longmans, Green and Company, 1928.

[62] A. Arya and E. Carter, "Structure, bonding, and adhesion at the $\mathrm{ZrC}$ (100)/Fe (110) interface from first principles," Surface science, 2004.

[63] D. Jiang and E. Carter, "Prediction of strong adhesion at the MoSi 2/Fe interface," Acta materialia, 2005.

[64] T. Hsieh and R. Balluffi, "Observations of roughening/de-faceting phase transitions in grain boundaries," Acta Metallurgica, vol. 37, pp. 2133-2139, Aug. 1989.

[65] E. T. Jaynes, "Information theory and statistical mechanics," Physical review, vol. 106, no. 4, p. 620, 1957.

[66] E. T. Jaynes, "Information theory and statistical mechanics. ii," Physical review, vol. 108, no. 2, p. 171, 1957.

[67] Y. Kulkarni, Coarse-graining of atomistic description at finite temperature. PhD thesis, California Institute of Technology, 2007.

[68] C. Villani, Topics in optimal transportation, vol. 58 of Graduate Studies in Mathematics. American Mathematical Society, Providence, RI, 2003.

[69] A. Romanov and T. Wagner, "On the universal misfit parameter at mismatched interfaces," Scripta materialia, vol. 45, no. 3, pp. 325-331, 2001.

[70] E. M. Stein and G. Weiss, Introduction to Fourier Analysis on Euclidean Spaces. PRINCETON University Press, 1971.

[71] Y. Kulkarni, Coarse-graining of atomistic description at finite temperature. PhD thesis, California Institute of Technology, 2007.

[72] Y. Shibuta, S. Takamoto, and T. Suzuki, "A molecular dynamics study of the energy and structure of the symmetric tilt boundary of iron," ISIJ international, 2008.

\section{Appendix A. $L^{2}$-control of the energy}

We proceed to show that the interatomic energy (12) corresponding to a thermalized atomic density is controled by the $L^{2}$ norm of the density. We take this bound as a convenient basis for the comparisons with 
experiment presented in Section 3.

Lemma 1. Let $f, g \in L_{\mathrm{loc}}^{1}\left(\mathbb{R}^{n}\right)$ be such that

$$
\frac{1}{c} \leq f, g \leq c \text { everywhere, }
$$

let $\theta \in C_{c}^{\infty}\left(\mathbb{R}^{n}\right)$ be radially symmetric and monotone, in the sense that $\theta(x)=h(|x|)$ with $h$ nonincreasing.

Then there is a bijective map $\varphi: \mathbb{R}^{n} \rightarrow \mathbb{R}^{n}$ such that

$$
\int_{\omega} f d x=\int_{\varphi(\omega)} g d x \quad \text { for all measurable sets } \omega
$$

and

$$
\int_{\mathbb{R}^{n}}|\varphi(x)-x|^{2}\left(\theta(x)+\theta(\varphi(x)) f(x) d x \leq C \int_{\mathbb{R}^{n}}|f(x)-g(x)|^{2} \theta(x) d x .\right.
$$

The constant $C$ may depend on $c, n$ and $\theta$.

Remark: The transport condition (A.2) is the same as $f(x)=g(\varphi(x)) \operatorname{det} D \varphi(x)$ a.e.

Proof. For $n=1$, using $\omega=(0, R)$ we see that $\varphi$ can be implicitly defined by

$$
\int_{0}^{R} f d x=\int_{0}^{\varphi(R)} g d x \text { for all } R>0 .
$$

Subtracting $\int_{0}^{R} g d x$

$$
\int_{0}^{R}(f-g) d x=\int_{R}^{\varphi(R)} g d x
$$

Since $g \geq 1 / c$, we obtain

$$
|\varphi(R)-R| \leq c \int_{0}^{R}|f-g| d x .
$$

By monotonicity, this gives

$$
|\varphi(R)-R| \theta^{1 / 2}(R) \leq c \int_{0}^{R}|f-g| \theta^{1 / 2} d x .
$$

By Hölder, $|\varphi(R)-R|^{2} \theta(R) \leq c R \int|f-g|^{2} \theta d x$ for all $R$. Integrating gives

$$
\int_{\mathbb{R}}|\varphi(x)-x|^{2} \theta(x) d x \leq C \int_{\mathbb{R}}|f-g|^{2}(x) \theta(x) d x,
$$

with $C=c^{2} \lambda^{2}$, where $\lambda$ is defined by $\theta \in C_{c}^{\infty}((-\lambda, \lambda))$. Since $f$ is bounded, this proves the estimate for the term with $\theta(x)$. In order to estimate the term with $\theta(\varphi(x))$, we proceed analogously. Subtracting $\int_{0}^{\varphi(R)} f d x$ from (A.4) gives

$$
\int_{\varphi(R)}^{R} f d x=\int_{0}^{\varphi(R)}(g-f) d x
$$

We have

$$
|\varphi(R)-R| \theta^{1 / 2}(\varphi(R)) \leq c \int_{0}^{\varphi(R)}|f-g| \theta^{1 / 2} d x
$$

and

$$
\int_{\mathbb{R}}|\varphi(x)-x|^{2} \theta(\varphi(x)) d x \leq C \int_{\mathbb{R}}|f-g|^{2}(x) \theta(x) d x .
$$

This concludes the proof for $n=1$. 
In dimension larger than 1 , we set $\varphi(r e)=\psi_{e}(r) e$, where, for every unit vector $e \in S^{n-1}, \psi_{e}:[0, \infty) \rightarrow$ $[0, \infty)$. This corresponds to setting $\varphi(x)=x \psi(x) /|x|$. We compute

$$
D \varphi=\frac{x}{|x|} \otimes D \psi+\frac{\operatorname{Id}|x|^{2}-x \otimes x}{|x|^{3}} \psi,
$$

which gives $\operatorname{det} D \varphi=\left(\partial_{r} \psi\right)(\psi / r)^{n-1}$. Therefore $\varphi$ is an admissible transport map if

$$
r^{n-1} f(r e)=g\left(\psi_{e}(r) e\right) \psi_{e}^{n-1}(r) \psi_{e}^{\prime}(r),
$$

for all $r$ and $e$, where we write $\psi_{e}^{\prime}=\partial_{r} \psi_{e}$. Integrating and changing variables to $s=\psi_{e}(r)$ gives

$$
\int_{0}^{R} r^{n-1} f(r e) d r=\int_{0}^{\psi_{e}(R)} g(s e) s^{n-1} d s
$$

We subtract $\int_{0}^{R} r^{n-1} g(r e) d r$ to obtain

$$
\int_{0}^{R} r^{n-1}(f-g)(r e) d r=\int_{R}^{\psi_{e}(R)} g(s e) s^{n-1} d s .
$$

Since $g \geq 1 / c$,

$$
\left|R^{n}-\psi_{e}(R)^{n}\right| \leq c \int_{0}^{R} r^{n-1}|f-g|(r e) d r .
$$

Using $R^{n}-\psi_{e}^{n}=\left(R-\psi_{e}\right) \sum_{i=0}^{n-1} R^{i} \psi_{e}^{n-1-i}$, we estimate

$$
\left|R-\psi_{e}(R)\right| \leq \frac{c}{R^{n-1}+\psi_{e}^{n-1}(R)} \int_{0}^{R} r^{n-1}|f-g|(r e) d r .
$$

Using, as above, monotonicity and Hölder,

$$
\left|R-\psi_{e}(R)\right|^{2} \theta(R e) \leq \frac{c^{2}}{\left(R^{n-1}+\psi_{e}^{n-1}(R)\right)^{2}}\left(\int_{0}^{R} r^{n-1}|f-g|^{2}(r e) \theta(r e) d r\right)\left(\int_{0}^{R} r^{n-1} d r\right) .
$$

The last integral gives $R^{n} / n$. We finally integrate over all $z \in B_{\lambda}$, and obtain

$$
\begin{aligned}
\int_{B_{\lambda}}|z-\varphi(z)|^{2} \theta(z) d z & =\int_{S^{n-1}} d \mathcal{H}^{n-1}(e) \int_{0}^{\lambda} d R R^{n-1}|R e-\varphi(R e)|^{2} \theta(R e) \\
& \leq \int_{S^{n-1}} d \mathcal{H}^{n-1}(e) \int_{0}^{\lambda} d R \frac{c^{2} R^{n} R^{n-1}}{n\left(R^{n-1}+\psi_{e}^{n-1}(R)\right)^{2}} \int_{0}^{R} r^{n-1}|f-g|^{2}(r e) \theta(r e) d r \\
& \leq C \int_{B_{\lambda}}|f-g|^{2}(z) \theta(z) d z .
\end{aligned}
$$

In this case $C=c \lambda^{2} / n$. To treat the term with $\theta(\varphi(x))$, as in the one-dimensional case we subtract $\int_{0}^{\psi_{e}(R)} r^{n-1} f(r e) d r$ from (A.14), obtain instead of (A.17) the estimate

$$
\left|R-\psi_{e}(R)\right| \leq \frac{c}{R^{n-1}+\psi_{e}^{n-1}(R)} \int_{0}^{\psi_{e}(R)} r^{n-1}|f-g|(r e) d r
$$

and continue as above. 


\begin{tabular}{|c|c|c|c|c|}
\hline Type & Orientation & Material & RMS Error & Predicted cusp locations \\
\hline FCC Tilt & [001] & $\mathrm{Cu}$ & $23 \%$ (LJ), 21\% (EAM) & $0^{\circ}, 53.1^{\circ}, 90^{\circ}$ \\
\hline FCC Tilt & {$[011]$} & $\mathrm{Cu}$ & $18 \%(\mathrm{LJ}), 27 \%(\mathrm{EAM})$ & $0^{\circ}, 70.5^{\circ}, 129.6^{\circ}, 90^{\circ}$ \\
\hline FCC Tilt & {$[111]$} & $\mathrm{Cu}$ & $22 \%$ (LJ), 26\% (EAM) & $0^{\circ}, 60^{\circ}$ \\
\hline FCC Tilt & {$[112]$} & $\mathrm{Cu}$ & $19 \%(\mathrm{LJ}), 27 \%(\mathrm{EAM})$ & $0^{\circ}, 63^{\circ}, 101.6^{\circ}$ \\
\hline FCC Tilt & {$[001]$} & $\mathrm{Au}$ & $25 \%(\mathrm{EAM})$ & $0^{\circ}, 53.1^{\circ}, 90^{\circ}$ \\
\hline FCC Tilt & {$[011]$} & $\mathrm{Au}$ & $20 \%(\mathrm{EAM})$ & $0^{\circ}, 70.5^{\circ}, 129.6^{\circ}, 90^{\circ}$ \\
\hline FCC Tilt & [111] & $\mathrm{Au}$ & $23 \%(\mathrm{EAM})$ & $0^{\circ}, 60^{\circ}$ \\
\hline FCC Tilt & {$[112]$} & $\mathrm{Au}$ & $23 \%$ (EAM) & $0^{\circ}, 63^{\circ}, 101.6^{\circ}$ \\
\hline BCC Tilt & {$[001]$} & Mo & $15 \%$ & $0^{\circ}, 37.0^{\circ}, 90^{\circ}$ \\
\hline BCC Tilt & {$[011]$} & Mo & $15 \%$ & $0^{\circ}, 50.5^{\circ}, 70.8^{\circ}, 109.6^{\circ}, 141.3^{\circ}, 180^{\circ}$ \\
\hline BCC Tilt & {$[111]$} & Mo & $16 \%$ & $0^{\circ}, 32.3^{\circ}, 90^{\circ}$ \\
\hline BCC Tilt & {$[112]$} & Mo & $9.6 \%$ & $0^{\circ}$ \\
\hline BCC Tilt & {$[001]$} & $\mathrm{Fe}$ & $31 \%$ & $0^{\circ}, 37.0^{\circ}, 90^{\circ}$ \\
\hline BCC Tilt & {$[011]$} & $\mathrm{Fe}$ & $21 \%$ & $0^{\circ}, 50.5^{\circ}, 70.8^{\circ}, 109.6^{\circ}, 141.3^{\circ}, 180^{\circ}$ \\
\hline BCC Tilt & [111] & $\mathrm{Fe}$ & $20 \%$ & $0^{\circ}, 32.3^{\circ}, 90^{\circ}$ \\
\hline BCC Tilt & {$[112]$} & $\mathrm{Fe}$ & $12 \%$ & $0^{\circ}$ \\
\hline FCC Twist & $(100)(100)$ & $\mathrm{Cu}$ & $33 \%$ & $0^{\circ}, 90^{\circ}$ \\
\hline FCC Twist & $(111)(111)$ & $\mathrm{Cu}$ & $127 \%$ & $0^{\circ}, 60^{\circ}$ \\
\hline FCC Twist & $(114)(011)$ & $\mathrm{Cu}$ & $17 \%$ & $0^{\circ}, 180^{\circ}$ \\
\hline FCC Twist & $(115)(111)$ & $\mathrm{Cu}$ & $3.5 \%$ & $0^{\circ}, 60^{\circ}$ \\
\hline FCC Twist & $(221)(001)$ & $\mathrm{Cu}$ & $7.1 \%$ & $0^{\circ}, 90^{\circ}$ \\
\hline FCC Twist & $(557)(113)$ & $\mathrm{Cu}$ & $13.4 \%$ & $0^{\circ}, 180^{\circ}$ \\
\hline FCC Twist & $(114)(011)$ & $\mathrm{Au}$ & $16.7 \%$ & $0^{\circ}, 180^{\circ}$ \\
\hline FCC Twist & $(115)(111)$ & $\mathrm{Au}$ & $6.1 \%$ & $0^{\circ}, 60^{\circ}$ \\
\hline FCC Twist & $(221)(001)$ & $\mathrm{Au}$ & $8.9 \%$ & $0^{\circ}, 90^{\circ}$ \\
\hline FCC Twist & $(557)(113)$ & $\mathrm{Au}$ & $14 \%$ & $0^{\circ}, 180^{\circ}$ \\
\hline BCC Twist & $(001)(001)$ & $\mathrm{Fe}$ & $14 \%$ & $0^{\circ}, 90^{\circ}$ \\
\hline BCC Twist & $(011)(011)$ & $\mathrm{Fe}$ & $57 \%$ & $0^{\circ}, 71^{\circ}, 110^{\circ}, 180^{\circ}$ \\
\hline BCC Twist & $(031)(031)$ & $\mathrm{Fe}$ & $17 \%$ & $0^{\circ}, 180^{\circ}$ \\
\hline BCC Twist & $(111)(111)$ & $\mathrm{Fe}$ & $25 \%$ & $0^{\circ}, 60^{\circ}$ \\
\hline BCC Twist & $(112)(112)$ & $\mathrm{Fe}$ & $17 \%$ & $0^{\circ}, 180^{\circ}$ \\
\hline BCC Twist & $(114)(011)$ & Mo & $3.3 \%$ & $0^{\circ}, 70.1^{\circ}, 109.7^{\circ}, 180^{\circ}$ \\
\hline BCC Twist & $(115)(111)$ & Mo & $22 \%$ & $0^{\circ}, 60^{\circ}$ \\
\hline BCC Twist & $(158)(031)$ & Mo & $21 \%$ & \\
\hline BCC Twist & $(221)(001)$ & Mo & $9.8 \%$ & $0^{\circ}, 90^{\circ}$ \\
\hline BCC Twist & $(552)(112)$ & Mo & $12 \%$ & $0^{\circ}, 180^{\circ}$ \\
\hline BCC Twist & $(114)(011)$ & $\mathrm{Fe}$ & $2.3 \%$ & $0^{\circ}, 70.1^{\circ}, 109.7^{\circ}, 180^{\circ}$ \\
\hline BCC Twist & $(115)(111)$ & $\mathrm{Fe}$ & $19 \%$ & $0^{\circ}, 60^{\circ}$ \\
\hline BCC Twist & $(158)(031)$ & $\mathrm{Fe}$ & $13 \%$ & \\
\hline BCC Twist & $(221)(001)$ & $\mathrm{Fe}$ & $13 \%$ & $0^{\circ}, 90^{\circ}$ \\
\hline BCC Twist & $(552)(112)$ & $\mathrm{Fe}$ & $5.8 \%$ & $0^{\circ}, 180^{\circ}$ \\
\hline
\end{tabular}

Table 3: Summary of results for FCC/BCC Tilt/Twist grain boundaries: RMS error and cusp locations predicted by the model. 